\title{
Thermodynamics of AdS black holes in Einstein-Scalar gravity
}

\section{H. Lü, ${ }^{a}$ C.N. Pope ${ }^{b, c}$ and Qiang Wen ${ }^{d}$}

\author{
${ }^{a}$ Department of Physics, Beijing Normal University, \\ Beijing 100875, China \\ ${ }^{b}$ George P. \& Cynthia Woods Mitchell Institute for Fundamental Physics and Astronomy, \\ Texas A\&M University, \\ College Station, TX 77843, U.S.A. \\ ${ }^{c}$ DAMTP, Centre for Mathematical Sciences, Cambridge University, \\ Wilberforce Road, Cambridge CB3 OWA, U.K. \\ ${ }^{d}$ Department of Physics, Renmin University of China, \\ Beijing 100872, China \\ E-mail: mrhonglu@gmail.com, pope@physics.tamu.edu, wen-q@ruc.edu.cn
}

ABSTRACT: We study the thermodynamics of $n$-dimensional static asymptotically AdS black holes in Einstein gravity coupled to a scalar field with a potential admitting a stationary point with an AdS vacuum. Such black holes with non-trivial scalar hair can exist provided that the mass-squared of the scalar field is negative, and above the BreitenlohnerFreedman bound. We use the Wald procedure to derive the first law of thermodynamics for these black holes, showing how the scalar hair (or "charge") contributes non-trivially in the expression. We show in general that a black hole mass can be deduced by isolating an integrable contribution to the (non-integrable) variation of the Hamiltonian arising in the Wald construction, and that this is consistent with the mass calculated using the renormalised holographic stress tensor and also, in those cases where it is defined, with the mass calculated using the conformal method of Ashtekar, Magnon and Das. Similar arguments can also be given for the smooth solitonic solutions in these theories. Neither the black hole nor the soliton solutions can be constructed explicitly, and we carry out a numerical analysis to demonstrate their existence and to provide approximate checks on some of our thermodynamic results.

KeYwords: Black Holes in String Theory, AdS-CFT Correspondence, Black Holes

ARXIV EPRINT: 1408.1514 


\section{Contents}

1 Introduction 1

2 Static solutions for Einstein-Scalar black holes $\quad 8$

3 Calculation of mass and the first law $\quad 9$

3.1 Derivation of the first law 9

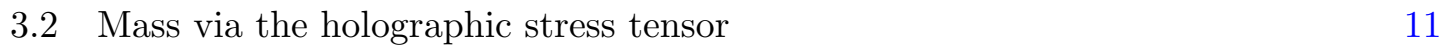

$\begin{array}{lll}3.3 & \text { AMD derivation of the mass } & 13\end{array}$

4 Asymptotics and thermodynamics for $0<\sigma<1 \quad 13$

5 Further examples outside $0<\sigma<1 \quad \mathbf{1 5}$

$\begin{array}{lll}5.1 \text { Special examples } & 16\end{array}$

$\begin{array}{ll}5.1 .1 n=4, \sigma=1 & 16\end{array}$

$\begin{array}{ll}5.1 .2 n=5, \sigma=1 & 17\end{array}$

$\begin{array}{lll}5.1 .3 n=5, \sigma=2 & 18\end{array}$

$\begin{array}{ll}5.1 .4 n=5, \sigma=\frac{5}{2} & 19\end{array}$

5.1.5 $n=7, \sigma=2 \quad 20$

5.1.6 The Breitenlohner-Freedman limit $\sigma=0 \quad 21$

$\begin{array}{lll}5.1 .7 & \text { Imaginary } \sigma & 22\end{array}$

6 Numerical analysis of black holes in gauged supergravities 23

6.1 Inner expansions 24

$\begin{array}{ll}6.2 n=4 \text { dimensions } & 25\end{array}$

$6.3 n=5$ dimensions 26

$6.4 n=6,7$ dimensions 27

$\begin{array}{llr}7 & \text { Conclusions } & 29\end{array}$

\section{Introduction}

The study of black hole thermodynamics has been one of the driving forces behind developments in general relativity and string theory in recent decades. These developments include techniques based on constructing Noether charges for deriving the first law of thermodynamics [1-3], and the construction of black hole solutions in a wide variety of gravity and supergravity theories. In this paper, we shall explore some subtleties arising in the first law of thermodynamics for black holes in Einstein gravity coupled to a scalar field, where there is a scalar potential that has a non-vanishing value at a stationary point. Such potentials typically arise in gauged supergravity theories, leading to the existence of 
black hole solutions that are asymptotic to anti-de Sitter (AdS) spacetime. The focus of our investigation will be the role of the parameter characterising the scalar "hair" in the first law of thermodynamics. For convenience we shall often refer to this parameter as a "scalar charge," although strictly speaking it is not a conserved quantity in the usual sense. Many properties of the scalar charges in asymptotic AdS backgrounds have been studied in literature; see, for example, [4-10].

We shall consider an $n$-dimensional theory of gravity coupled to a scalar field $\phi$, described by the Lagrangian

$$
\mathcal{L}=\sqrt{-g}\left(R-\frac{1}{2}(\partial \phi)^{2}-V(\phi)\right)
$$

The potential $V(\phi)$ will be assumed to have a stationary point at $\phi=0$, such that $V(0)$ is negative and the theory admits an AdS vacuum solution. We shall look for static spherically symmetric solutions that approach the AdS vacuum at large distance, with the ansatz

$$
d s^{2}=-h(r) d t^{2}+\frac{d r^{2}}{f(r)}+r^{2} d \Omega_{n-2}^{2}, \quad \phi=\phi(r),
$$

where $d \Omega_{n-2}^{2}$ is the metric on the unit $(n-2)$-sphere. We shall consider two different kinds of short-distance behaviour; either a black hole, for which $h(r)$ vanishes at some radius $r=r_{0}$ (the outer horizon), or else a smooth soliton, where $f(r)$ approaches 1 and $h(r)$ approaches a constant as $r$ goes to zero.

Even for the static spherically symmetric ansatz that we are considering in this paper, the equations of motion following from (1.1) are too complicated to admit explicit closedform solutions in general, and so the strategy for finding the black hole or soliton solutions has to depend on numerical analysis and computer integration of the equations. A convenient way to do this is first to obtain the general asymptotic forms of the scalar and metric functions at large $r$, and then to use short-distance expansions valid near the horizon (or at the origin, in the solitonic case) to set initial conditions for a numerical integration out to large distance. If such a technique were applied to finding asymptotically flat black holes with massive scalars, it would require a very delicate matching at large distance because the asymptotic forms of the general large- $r$ solutions would include terms with unacceptable exponentially-growing behaviour. By contrast, in the asymptotically-AdS case the general large- $r$ solutions are all compatible with the AdS asymptotics, provided that the mass of the scalar field lies in an appropriate range. This then means that having set initial conditions for a black hole near the horizon, the scalar and metric functions essentially cannot fail to integrate out to have acceptable large- $r$ behaviour. Thus, while asymptotically-flat black holes with massive scalar hair cannot arise (and indeed are ruled out by no-hair theorems), asymptotically-AdS black holes, or solitons, with scalar hair are commonplace, provided the mass of the scalar field lies in an appropriate range.

Later in the paper we shall study the near-horizon structure of the black-hole solutions. For now, it suffices to record that the general such near-horizon solutions turn out to be characterised by two non-trivial parameters, which may be thought of as the horizon radius $r_{0}$ and a scalar parameter $\phi_{0}=\phi\left(r_{0}\right)$ (the value of the scalar field on the horizon). On 
the other hand, the large- $r$ solutions are characterised by three parameters, which we may think of as a "mass parameter" $\alpha$, which is the coefficient of the $r^{-(n-3)}$ term in the large- $r$ expansion of the metric function $h(r)$,

$$
h(r)=r^{2} \ell^{-2}+\cdots+\frac{\alpha}{r^{n-3}}+\cdots,
$$

and two coefficients, $\phi_{1}$ and $\phi_{2}$, characterising the leading-order terms in the two independent solutions of $\phi(r)$ at large $r$. When we eventually match the near-horizon expansion to the large- $r$ expansion, the three parameters in the asymptotic expansion will be determined as functions of the two non-trivial parameters of the near-horizon solution. Equivalently, one may view two of the asymptotic parameters as being independent, with the third being determined in terms of these. Specifically, we shall refer to $\phi_{2}$, the coefficient of the leading-order term in the faster-falling of the two scalar solutions, as the "scalar charge." For now, we may proceed by just considering the large- $r$ expansion, since this is what is needed in order to investigate the contribution of the scalar charge to the first law of thermodynamics. We just need to bear in mind that eventually, the details of the black hole solutions will impose one relation between the three parameters $\alpha, \phi_{1}$ and $\phi_{2}$.

The situation is similar in the case of soliton solutions, except that now the general short-distance solution has just the single non-trivial parameter $\phi_{0}$. This then implies that ifor solitons there will be two relations among the three asymptotic parameters $\alpha, \phi_{1}$ and $\phi_{2}$.

Our aims in this paper are to demonstrate the existence of the static spherically symmetric black holes and solitons with scalar hair; to derive a first law of black hole dynamics, and to discuss the notion of an energy function, or "mass," for the black hole solutions. The first law of black hole dynamics for a spherically-symmetric black hole in pure Einstein gravity takes the form $d M=\kappa d A /(8 \pi)$, where $\kappa$ is the surface gravity and $A$ is the area of the event horizon. As is well known, with the Hawking temperature being related to the surface gravity by $T=\kappa /(2 \pi)$, and the Bekenstein-Hawking entropy given by $S=A / 4$, the first law of black hole dynamics becomes the first law of thermodynamics $d M=T d S$ for the spherically-symmetric black holes of pure Einstein gravity. In what follows, we shall typically use the language and the variables of the thermodynamic first law in our discussion, but it should be borne in mind that we are really just considering the purely classical dynamics of the black hole solutions, with $T$ meaning $\kappa /(2 \pi)$ and $S$ meaning $A / 4$.

To discuss the first law for the Einstein-Scalar black holes and solitons, we adopt a general procedure described in [1-3]. This involves considering an infinitesimal variation in a family of solutions admitting a timelike Killing vector, and deriving a closed $(n-$ 2 )-form whose integral $\delta \mathcal{H}$ over a bounding spacelike surface is therefore independent of deformations of the surface. In particular, this means

$$
\delta \mathcal{H}_{\infty}=\delta \mathcal{H}_{H^{+}}
$$

where $\delta \mathcal{H}_{\infty}$ is evaluated on the sphere at infinity and $\delta \mathcal{H}_{H^{+}}$is evaluated on the outer horizon of the black hole.

In his discussions Wald has referred to two somewhat different possible viewpoints one make take, in regard to the infinitesimal variations that one considers in the derivation. 
In the first of these, called the "physical states" viewpoint, one considers a variation that results from an actual physical process under which the black hole evolves from an initial to a nearby final stationary black hole solution. In the second approach, referred to as the "equilibrium states" viewpoint, one simply considers the change that results from making arbitrary infinitesimal variations of all the parameters characterising the solution space for the black holes in the theory under consideration. We should stress at this point that in all our discussions we shall be adopting the second, equilibrium states, viewpoint.

As we mentioned earlier, the spherically-symmetric static black hole solutions of the Einstein-Scalar theories we are considering are characterised by two independent non-trivial parameters, which we may think of as the mass parameter $\alpha$ and the scalar "charge" parameter $\phi_{2}$. It is then of interest to seek a generalisation of the first law $d M=T d S$ of pure Einstein theory, for the two-parameter spherically-symmetric black holes of the Einstein-Scalar theories. Such a first law would cerainly require more than just the term $T d S$ on the right-hand side, since one can easily verify, as we shall see later, that $T d S$ by itself is not an exact form in the parameter space of the solutions, if one considers general infinitesimal variations of the two independent parameters in the black hole solutions.

For the static Einstein-scalar black holes we are considering in this paper, we find that

$$
\delta \mathcal{H}_{H^{+}}=T \delta S,
$$

where $T=\kappa /(2 \pi)$ is the Hawking temperature and $S=\frac{1}{4} A$, one quarter of the horizon area, is the Bekenstein-Hawking entropy. At infinity we find that

$$
\delta \mathcal{H}_{\infty}=\delta E+\left(c_{1} \phi_{2} \delta \phi_{1}-c_{2} \phi_{1} \delta \phi_{2}\right)
$$

where $c_{1}$ and $c_{2}$ are constants that are characteristic of the spacetime dimension and the mass of the scalar field. Note that $c_{2} \neq-c_{1}$, and so the contribution $\left(c_{1} \phi_{2} \delta \phi_{1}-c_{2} \phi_{1} \delta \phi_{2}\right)$ is not integrable (unless there is a functional relation of the form $\phi_{1}=\phi_{1}\left(\phi_{2}\right)$ between $\phi_{1}$ and $\phi_{2}$ ). The variation $\delta E$ appearing in (1.6), on the other hand, is integrable; it takes the form

$$
\delta E=\frac{\omega_{n-2}}{16 \pi}\left[-(n-2) \delta \alpha+\delta K\left(\phi_{1}, \phi_{2}\right)\right]
$$

where $\omega_{n-2}$ is the volume of the unit $(n-2)$ sphere and $K\left(\phi_{1}, \phi_{2}\right)$ is a calculable function of $\phi_{1}$ and $\phi_{2}$ that depends on the spacetime dimension and the precise form of the scalar potential $V(\phi)$ (including, in particular, the mass of the scalar field). Integrating (1.7) gives

$$
E=\frac{\omega_{n-2}}{16 \pi}\left[-(n-2) \alpha+K\left(\phi_{1}, \phi_{2}\right)\right] .
$$

By writing $\delta \mathcal{H}_{\infty}$ in the form (1.6), we have separated off its non-integrable portion, which is bilinear in $\phi_{i}$ and $\delta \phi_{j}$, and written the integrable remainder as the variation of the function $E$ given in (1.8). This decomposition is not unique, but the ambiguity is restricted to the freedom to add a constant multiple of $\phi_{1} \phi_{2}$ to $K$, leading to the redefined quantities

$$
K^{\prime}=K+\lambda \phi_{1} \phi_{2}, \quad c_{1}^{\prime}=c_{1}-\frac{\omega_{n-2} \lambda}{16 \pi}, \quad c_{2}^{\prime}=c_{2}+\frac{\omega_{n-2} \lambda}{16 \pi},
$$

and the associated redefinition of $E$, where $\lambda$ is any constant. 
At this point we have obtained a function $E$ that depends on the mass parameter $\alpha$ and the scalar "charge" $\phi_{2}$ (with $\phi_{1}$ being a function of $\alpha$ and $\phi_{2}$.) Obviously $E$ has the dimensions of energy or mass, and so it is natural to enquire whether it is related to any other known definition of a mass for an asymptotically-AdS black hole.

As we shall discuss in detail in this paper, the function $E$ can in fact be associated with the mass of the black hole, and so from (1.4), (1.5) and (1.6) we obtain the first law of thermodynamics for the Einstein-Scalar black holes, in the form

$$
d E=T d S-\left(c_{1} \phi_{2} d \phi_{1}-c_{2} \phi_{1} d \phi_{2}\right)
$$

Since all the quantities in (1.10) ultimately depend on the two non-trivial parameters $\alpha$ and $\phi_{2}$ that characterise the black hole solutions, we can interpret the first law as a statement of how the entropy changes under an infinitesimal variation of the mass and the scalar charge.

We could in fact take the calculation of $E$ described above as a definition of the mass, or energy, of the Einstein-Scalar black hole. The ambiguity associated with the redefinition (1.9) corresponds to the freedom to make a Legendre transformation from one type of energy variable to another, as one can always do for thermodynamic systems. (As, for example, in the transformation from the internal energy $U$ satisfying $d U=T d S+\cdots$ to the Gibbs free energy $G=U-T S+\cdots$ satisfying $d G=-S d T+\cdots$.) A unique definition of the energy function is pinned down by specifying the precise form of the first law; for example, by choosing $\lambda$ so that $c_{1}^{\prime}=0$ in (1.9).

We can give a more concrete interpretation for the quantity $E$ by using some independent procedure to compute the mass of the black holes. We shall consider two methods in this paper. The first, which seems to be the most reliable, is by using the AdS/CFT correspondence to calculate the renormalised stress tensor $T_{\alpha \beta}$ of the boundary conformal field theory, and then interpreting the appropriate integral of $T_{00}$ as the mass [11-15]. We may also compute the mass using the AMD conformal procedure developed by Ashtekar, Magnon and Das [16, 17], involving the integration of a certain electric component of the Weyl tensor over the spherical boundary at infinity. This works well provided the metric approaches AdS sufficiently rapidly, and we find for our solutions that the two approaches yield consistent results under these circumstances. ${ }^{1}$

We shall see that the quantity $E$ arising from the Wald derivation of the first law of thermodynamics is in fact consistent with the mass calculated using the renormalised holographic stress tensor. In certain cases there are ambiguities in the calculation of the holographic mass, including the one alluded to previously. These ambiguities amount to nothing more than the freedom to make a Legendre transformation from one energy function to another.

The variation $\delta \mathcal{H}_{\infty}$ in (1.6) is not integrable unless $\phi_{1}$ is a specific function of $\phi_{2}$ alone, whereas, by contrast, in the scalar black hole solutions $\phi_{1}$ is a function of $\phi_{2}$ and $E$. This means that as one varies the two independent parameters $\phi_{2}$ and $E$ one is effectively

\footnotetext{
${ }^{1} \mathrm{~A}$ recent discussion of the calculation of the AMD mass in the context of AdS black holes with scalar hair can be found in [18]. See also [19] for the calculation of the AMD mass in a variety of charged rotating AdS black holes in gauged supergravities.
} 
changing the boundary conditions on the scalar field at infinity. (We remind the reader that we are adopting the "equilibrium states" viewpoint in our discussions, in which arbitrary variations in the parameters in the solution are considered.) The non-integrability of $\delta \mathcal{H}_{\infty}$ is symptomatic of the existence of a non-trivial symplectic flux at infinity [1,2], which in our case would be proportional to the second variation $g^{2}\left(\delta_{(1)} \phi_{1} \wedge \delta_{(2)} \phi_{2}-\delta_{(2)} \phi_{1} \wedge \delta_{(1)} \phi_{2}\right)$ in field space. This has the implication that there does not exist a universal Hamiltonian $\mathcal{H}_{\infty}$ for the entire class of scalar black hole solutions, and so one does not have an energy function that is conserved for the entire class of solutions. Nonetheless, for any specific solution we can define the boundary condition on the scalar field to be the one satisfied by that particular black hole. The mass for this black hole can be calculated, without appeal to a variational calculation of $\delta \mathcal{H}_{\infty}$, via the computation of the holographic stress tensor. By this means we can define a mass for each black hole in the two-parameter family that is characterised by $\phi_{2}$ and $E$. Equation (1.4) then gives a mathematically valid statement of how the entropy $S\left(\phi_{2}, E\right)$ varies under infinitesimal changes in $\phi_{2}$ and $E$, regardless of the fact that such changes will in general move the solution to a new configuration for which the boundary condition on $\phi$ has altered.

It has been observed in previous discussions (see, for example, [8], and, more recently, in [18]) that if one is considering the case of a solution where $\phi_{1}$ is determined as a function of $\phi_{2}$ alone, then one can always integrate the full asymptotic quantity $\delta \mathcal{H}_{\infty}$ in (1.6) and take it as a definition of the mass. Indeed, one could do this for the solitonic solutions of the Einstein-Scalar theory since, as we remarked above, the solutions are characterised by the single non-trivial parameter $\phi_{0}$ at the origin, and therefore $\alpha, \phi_{1}$ and $\phi_{2}$ in the asymptotic solutions are all functions of just $\phi_{0}$, and hence for the solitons we can view $\phi_{1}$ as a function of $\phi_{2} .{ }^{2}$ However, it is important to stress that one cannot do this for the black hole solutions, since now $\alpha, \phi_{1}$ and $\phi_{2}$ are functions of the two non-trivial parameters $r_{0}$ and $\phi_{0}$ on the horizon. One can therefore view $\phi_{1}$ as a function of $\phi_{2}$ and $\alpha$, but not as a function of $\phi_{2}$ alone. Thus one cannot integrate the entire quantity $\delta \mathcal{H}_{\infty}$ to obtain a "mass" in this case. It is for this reason that we argued that one has to separate off the non-integrable terms involving $\phi_{2} \delta \phi_{1}$ and $\phi_{1} \delta \phi_{2}$ in (1.6), and interpret only the integrable remainder $\delta E$ as the variation of a mass. In this viewpoint, the non-integrable terms must then be interpreted as a distinct additional contribution in the first law of thermodynamics. In other words, the solitonic solutions, where one could choose to integrate up the $\phi_{2} \delta \phi_{1}$ and $\phi_{1} \delta \phi_{2}$ terms and absorb them into a Legendre transformed energy function, are very special, and not representative of the more general situation with black holes, where there are independent mass and scalar charge parameters.

Some of the above considerations came rather strikingly to the fore recently, with the construction of a dyonic charged black hole in a certain four-dimensional gauged supergrav-

\footnotetext{
${ }^{2}$ The mass defined in this way would, of course, depend upon the functional dependence of $\phi_{1}$ upon $\phi_{2}$ that followed as a consequence of the equations of motion in the interior. By contrast, the mass calculated using the AMD proceedure or the holographic renormalised stress tensor depends only on certain coefficients in the asymptotic expansions, in an algebraic and local fashion. How these coefficients are constrained by the interior behaviour, be it a soliton (smooth), a black hole (with a horizon), or an object with naked singularity, should not affect the definition of the mass.
} 
ity theory [20]. (See also [21].) This theory comprises Einstein-Scalar gravity coupled to a Maxwell field, with a very specific scalar potential and scalar coupling to the Maxwell field. The novelty of this solution lies in the fact that it is actually fully explicit and expressible in closed form, and yet, it exhibits precisely the kind of phenomenon that we have discussed above, in which the scalar field contributes a non-trivial additional term in the first law of thermodynamics. The black holes in [20] depend on three non-trivial parameters, namely the mass $M$, the electric charge $Q$ and the magnetic charge $P$. The asymptotic scalar parameters $\phi_{1}$ and $\phi_{2}$ are specific functions of $M, Q$ and $P$, and one cannot simply integrate up the $\phi_{2} \delta \phi_{1}$ and $\phi_{1} \delta \phi_{2}$ terms in the first law [20]. The solutions in [20], which are fully explicit, are not in fact the most general static spherically-symmetric black holes in the theory; there will actually exist four-parameter solutions in which the scalar charge can be independently specified. (These would be charged analogues of the Einstein-Scalar black holes we are considering in this paper.) However, as with the simpler case of the Einstein-Scalar system we are considering here, one cannot obtain the four-parameter black hole solutions explicitly. They would, however, provide further examples where one could not integrate up the contribution from the scalar charge in the variation $\delta \mathcal{H}_{\infty}$.

It is instructive to compare the situation for black holes in the Einstein-Scalar system to that for Reissner-Nordström black holes in the Einstein-Maxwell theory. A derivation of the first law using the Wald formalism was presented in [22], for a gauge choice where the vector potential $A$ vanishes asymptotically at infinity. In this gauge, one has

$$
\delta \mathcal{H}_{H^{+}}=T \delta S+\Phi \delta Q, \quad \delta \mathcal{H}_{\infty}=\delta M
$$

and so $\delta \mathcal{H}_{\infty}=\delta \mathcal{H}_{H^{+}}$leads to the standard first law [22], and furthermore $\delta \mathcal{H}_{\infty}$ is integrable. In the context of the AdS/CFT correspondence, however, it is customary instead to make a gauge choice for which $A$ vanishes on the horizon, and consequently $A_{0} \sim \Phi+Q / r$ in the asymptotic region. In this gauge choice, $\Phi$ acquires a physical interpretation as a chemical potential in the boundary field theory. We then have

$$
\delta \mathcal{H}_{H^{+}}=T \delta S, \quad \delta \mathcal{H}_{\infty}=\delta M-\Phi \delta Q
$$

with $\delta \mathcal{H}_{\infty}=\delta \mathcal{H}_{H^{+}}$again leading to the same first law. However, for this gauge choice $\delta \mathcal{H}_{\infty}$ is no longer integrable, just as we have seen in the case of Einstein-Scalar black holes. The gauge choice where $A$ vanishes on the horizon is also a more natural one if one views the Maxwell field as a massless limiting case of a more general class of massive Proca fields, since for the Einstein-Proca system the equations of motion imply that the Proca field $A$ must vanish on the horizon. (See [23] for a detailed discussion of the thermodynamics of Einstein-Proca AdS black holes.)

The organisation of the rest of this paper is as follows. In section 2, we set up our notation and conventions for the Einstein-Scalar theory, and we derive the equations for motion for the fields in the static, spherically-symmetric, ansatz for black hole and soliton solutions. Section 3 contains a derivation of the first law of thermodynamics for the black holes and solitons, and also the derivation of their mass, using the holographic stress tensor and also using the conformal procedure developed by Ashtekar, Magnon and Das. 
In section 4, we discuss the asymptotic forms of the solutions at large distance, focusing especially on the case where the mass of the scalar field lies in a particular range for which a generic discussion of the large- $r$ expansions can easily be given. We also use the results from section 3 to calculate the masses of the black holes for this class of solutions, and to give an explicit derivation of the first law of thermodynamics. ${ }^{3}$ In section 5 we study a variety of cases where the mass of the scalar field lies outside the generic range discussed in section 4 , in some of which additional complications or subtleties arise. In section 6 we turn to a numerical study of the black hole and soliton solutions. This includes a derivation of the inner expansions for the solutions near to the horizon or the origin respectively, which we then use in order to set initial data for numerical integrations out to large distances. We use some of the numerical results in order to obtain approximate confirmation of our results for the first law.

\section{Static solutions for Einstein-Scalar black holes}

The equations of motion following from the Lagrangian (1.1) are

$$
\square \phi=\frac{\partial V}{\partial \phi}, \quad E_{\mu \nu} \equiv R_{\mu \nu}-\frac{1}{2} \partial_{\mu} \phi \partial_{\nu} \phi-\frac{1}{n-2} V g_{\mu \nu}=0 .
$$

The potential $V(\phi)$ will be assumed to have a stationary point at $\phi=0$, at which the potential is some negative constant. It will be convenient to take

$$
V(0)=-(n-1)(n-2) \ell^{-2},
$$

which therefore implies that the anti-de Sitter spacetime

$$
d s^{2}=-\left(g^{2} r^{2}+1\right) d t^{2}+\left(g^{2} r^{2}+1\right)^{-1} d r^{2}+r^{2} d \Omega_{n-2}^{2}
$$

is a solution, where $d \Omega_{n-2}^{2}$ is the metric on the unit $(n-2)$-sphere, and where the scalar field $\phi$ is set to zero. Here, and in much of the remainder of the paper, it is convenient to define the constant $g$ (like a gauge-coupling constant in gauged supergravity) by

$$
g=\frac{1}{\ell} .
$$

We shall assume that the scalar potential admits a Taylor expansion of the form

$$
V=-(n-1)(n-2) g^{2}+\frac{1}{2} m^{2} \phi^{2}+\gamma_{3} \phi^{3}+\gamma_{4} \phi^{4}+\cdots .
$$

The parameter $m$ is the mass of the scalar field.

The linearised equation for the scalar field around the AdS background is $\left(\square-m^{2}\right) \phi=$ 0 , for which the general static spherically-symmetric solution is

$$
\begin{aligned}
\phi= & \frac{c_{1}}{r^{(n-1-\sigma) / 2}}{ }_{2} F_{1}\left[\frac{1}{4}(5-n-\sigma), \frac{1}{4}(n-1-\sigma) ; 1-\frac{1}{2} \sigma ;-\frac{\ell^{2}}{r^{2}}\right] \\
& \frac{c_{2}}{r^{(n-1+\sigma) / 2}}{ }_{2} F_{1}\left[\frac{1}{4}(5-n+\sigma), \frac{1}{4}(n-1+\sigma) ; 1+\frac{1}{2} \sigma ;-\frac{\ell^{2}}{r^{2}}\right],
\end{aligned}
$$

\footnotetext{
${ }^{3}$ Our focus throughout is on spherically-symmetric black holes in dimesions $n \geq 4$. One could also consider more general black holes, and black holes in three dimensional Einstein-Scalar theories, which have been studied, for example, in [24].
} 
where

$$
\sigma=\sqrt{4 \ell^{2} m^{2}+(n-1)^{2}} .
$$

More general time-dependent modes will have real frequencies provided that $\sigma$ is real, and this implies that the modes are non-tachyonic provided that

$$
m^{2} \geq m_{\mathrm{BF}}^{2}=-\frac{1}{4}(n-1)^{2} \ell^{-2},
$$

where the (negative) mass-squared $m_{\mathrm{BF}}^{2}$ is known as the Breitenlohner-Freedman bound. The scalar fields in supergravity theories commonly have negative values of mass-squared, lying within the allowed Breitenlohner-Freedman range.

As well as AdS itself, the theory described by (1.1) also admits black hole solutions, such as Schwarzschild-AdS, for which the scalar field continues to vanish. However, we may also consider more general black-hole solutions, where the scalar field is excited too. We shall consider only static, spherically symmetric, black holes in this paper, for which the metric and scalar field may be assumed to take the form (1.2). The non-vanishing components of the Ricci tensor are given by

$$
\begin{aligned}
& R_{t t}=h f\left(\frac{h^{\prime \prime}}{2 h}-\frac{h^{\prime 2}}{4 h^{2}}+\frac{h^{\prime} f^{\prime}}{4 h f}+\frac{(n-2) h^{\prime}}{2 r h}\right), \\
& R_{r r}=-\frac{h^{\prime \prime}}{2 h}+\frac{h^{\prime 2}}{4 h^{2}}-\frac{h^{\prime} f^{\prime}}{4 h f}-\frac{(n-2) f^{\prime}}{2 r^{2} f}, \\
& R_{i j}=\left((n-3)-\frac{r(h f)^{\prime}}{2 h}-(n-3) f\right) \tilde{g}_{i j},
\end{aligned}
$$

where $\tilde{g}_{i j}$ is the metric on the unit $(n-2)$-sphere. The equations of motion implied by (2.1) can be taken to be

$$
\begin{aligned}
& \frac{h^{\prime \prime}}{h}-\frac{h^{2}}{2 h^{2}}+\frac{f^{\prime} h^{\prime}}{2 f h}+\frac{(n-3) h^{\prime}}{r h}-\frac{f^{\prime}}{r f}-\frac{2(n-3)(f-1)}{r^{2} f}=0, \\
& \phi^{\prime 2}=\frac{(n-2)\left(f h^{\prime}-h f^{\prime}\right)}{r f h}, \\
& \frac{f h^{\prime \prime}}{h}-\frac{f h^{\prime 2}}{2 h^{2}}+\frac{f^{\prime} h^{\prime}}{2 h}+\frac{(n-1) f h^{\prime}}{r h}+\frac{f^{\prime}}{r}+\frac{2(n-3)(f-1)}{r^{2}}+\frac{4 V}{n-2}=0 .
\end{aligned}
$$

Note that the first two equations, coming from $E_{t}{ }^{t}-E_{i}{ }^{i}=0$ and $E_{t}{ }^{t}-E_{r}{ }^{r}=0$, do not involve the potential $V(\phi)$.

\section{Calculation of mass and the first law}

\subsection{Derivation of the first law}

We adopt the general procedure developed by Wald [1] in order to derive the first law of thermodynamics for the Einstein-Scalar black holes. Specifically, we shall follow rather closely the derivation that was presented in [22] for obtaining the first law for the EinsteinMaxwell system, adapting it appropriately for our Einstein-Scalar case. For further specific 
details of the derivation for Einstein gravity coupled to a scalar field, we refer to some earlier work in [20] and [10]. The procedure involves considering the variation of the parameters in an $n$-dimensional solution, and constructing a closed $(n-2)$-form $\left(\delta Q-i_{\xi} \Theta\right)$ where $\xi$ is any Killing vector. Taking $\xi=\partial / \partial t$ and applying this to the theory (1.1) with the static solutions of the form (1.2), one finds that the integral of $\left(\delta Q-i_{\xi} \Theta\right)$ over any $S^{n-2}$ surface at constant $t$ and $r$ gives a quantity $\delta \mathcal{H}$ that is independent of $r$. Thus in particular we have (1.4), where the two quantities are evaluated at infinity and on the outer horizon.

In detail, the Wald procedure [1] involves first writing the variation of a Lagrangian $n$-form under a general diffeomorphism as $\delta L=E(\Phi) \delta \Phi+d \Theta(\Phi, \delta \Phi)$, where the fields $\Phi$ transform as $\delta \Phi=\mathcal{L}_{\xi} \Phi$, and where $\mathcal{L}_{\xi}$ is the Lie derivative with respect to the diffeomorphism parameter $\xi$, and $E(\Phi)=0$ are the equations of motion. Since one also has $\delta L=\mathcal{L}_{\xi} L$, and $\mathcal{L}_{\xi}=d i_{\xi}+i_{\xi} d$, it follows that

$$
\Theta\left(\Phi, \mathcal{L}_{\xi} \Phi\right)-i_{\xi} L=d Q
$$

where $Q$ is an $(n-2)$ form, and $i_{\xi}$ acting on any $p$-form gives the $(p-1)$-form obtained by contraction with $\xi$. One now makes a variation of the parameters in the solution, giving

$$
d \delta Q=\delta \Theta-i_{\xi} \delta L=\delta \Theta-i_{\xi} d \Theta=\delta \Theta-\mathcal{L}_{\xi} \Theta+d i_{\xi} \Theta,
$$

and then takes $\xi$ to be a Killing vector, for which $\mathcal{L}_{\xi} \Theta=0$ and $\delta \Theta=\delta \Theta\left(\Phi, \mathcal{L}_{\xi} \Phi\right)=0$ (since $\left.\mathcal{L}_{\xi} \Phi=0\right)$. Thus $\left.d\left(\delta Q-i_{\xi} \Theta\right)\right)=0$, implying that

$$
\delta \mathcal{H} \equiv \int\left(\delta Q-i_{\xi} \Theta\right)
$$

is independent of deformations of the closed $(n-2)$-dimensional surface over which it is integrated. For the ansatz (1.2) in the Einstein-Scalar theory, and taking $\xi$ to be the timelike Killing vector $\partial / \partial t$, one finds $[10,20]$

$$
\begin{aligned}
Q & =\frac{1}{16 \pi} r^{n-2} h^{\prime} \sqrt{\frac{f}{h}} \Omega_{n-2}, \\
i_{\xi} \Theta & =\frac{1}{16 \pi} r^{n-2}\left[\delta\left(h^{\prime} \sqrt{\frac{f}{h}}\right)+\frac{n-2}{r} \sqrt{\frac{h}{f}} \delta f+\sqrt{f h} \phi^{\prime} \delta \phi\right] \Omega_{n-2},
\end{aligned}
$$

where $\Omega_{n-2}$ is the volume form on the unit $(n-2)$-sphere, and hence at radius $r$ one has

$$
\delta \mathcal{H}=-\frac{\omega_{n-2}}{16 \pi} r^{n-2} \sqrt{\frac{h}{f}}\left[\frac{n-2}{r} \delta f+f \phi^{\prime} \delta \phi\right],
$$

where $\omega_{n-2}$ is the volume of the unit $S^{n-2}$. As we shall see below, $\delta \mathcal{H}_{\infty}$ turns out to be equal to be the variation $\delta E$ of a function $E$ of the independent asymptotic parameters of the solution plus a non-integrable contribution involving the variation of the coefficients $\phi_{1}$ and $\phi_{2}$ in the asymptotic expansion of the scalar field. The function $E$ has an interpretation as the mass of the black hole. On the other hand, $\delta \mathcal{H}$ can be evaluated on the horizon by considering the near-horizon form of the metric (1.2), for which we shall have

$$
h(r)=\left(r-r_{0}\right) h^{\prime}\left(r_{0}\right)+\cdots, \quad f(r)=\left(r-r_{0}\right) f^{\prime}\left(r_{0}\right)+\cdots,
$$


where $r_{0}$ is the horizon radius. Thus $\left.\delta f\right|_{r=r_{0}}=-\delta r_{0} f^{\prime}\left(r_{0}\right)$ and so

$$
\delta \mathcal{H}_{H^{+}}=\frac{(n-2) \omega_{n-2}}{16 \pi} \sqrt{f^{\prime}\left(r_{0}\right) h^{\prime}\left(r_{0}\right)} r_{0}^{n-3} \delta r_{0}=T \delta S,
$$

since $T=(4 \pi)^{-1} \sqrt{f^{\prime}\left(r_{0}\right) h^{\prime}\left(r_{0}\right)}$ and $(n-2) \omega_{n-2} r_{0}^{n-3} \delta r_{0}=\delta\left(r_{0}^{n-2} \omega_{n-2}\right)=\delta A=4 \delta S$. Thus (1.4) gives us the first law of thermodynamics for the Einstein-Scalar black holes.

\subsection{Mass via the holographic stress tensor}

The mass of the Einstein-Scalar black holes can be calculated using standard holographic techniques. That is to say, we calculate the renormalised stress tensor $T_{\alpha \beta}$ for the dual boundary theory that is related to the bulk theory via the AdS/CFT correspondence. Integrating the component $T_{00}$ over the spatial $S^{n-2}$ boundary at infinity gives the mass of the black hole. The renormalisation is achieved by adding appropriate boundary terms and counterterms to the bulk action, and $T_{\alpha \beta}$ is then obtained by evaluating the variation of the total action with respect to the boundary metric. In general, the counterterms in the gravitational sector will be certain invariant polynomials built from the boundary curvature tensor and its covariant derivatives. The first few such terms, sufficient for renormalising the stress tensor in some of the lower spacetime dimensions, can be found in the extensive literature on the subject. In our case, since we are focusing our attention on static and spherically-symmetric configurations, the contribution to $T_{00}$ from the gravitational counterterm at a given order, corresponding to a curvature polynomial of degree $p$, will necessarily take the form of a constant coefficient divided by $r^{2 p}$. Each of these constant coefficients will have a universal (dimension-dependent) value, independent of the parameters of the specific solution, which is uniquely determined by the requirement that it remove the corresponding divergence in the stress tensor for the pure AdS background. This enables us to perform the renormalisation in arbitrarily high dimensions without needing to know the detailed and complicated expressions for the general curvature counterterms that arise as one looks at higher dimensions.

The bulk Lagrangian, and the boundary and counterterms in the gravitational sector, are given by [13-15]

$$
\begin{aligned}
& \mathcal{L}_{\text {bulk }}= \frac{1}{16 \pi G} \sqrt{-g}\left[R-\frac{1}{2}(\partial \phi)^{2}-(n-1) \ell^{-2}\right], \\
& \mathcal{L}_{\text {surf }}=-\frac{1}{8 \pi G} \sqrt{-h} K \\
& \mathcal{L}_{\text {ct }}=\frac{1}{16 \pi G} \sqrt{-h}\left[-\frac{2(n-2)}{\ell}+\frac{\ell}{(n-3)} \mathcal{R}\right. \\
&\left.\quad+\frac{\ell^{3}}{(n-5)(n-3)^{2}}\left(\mathcal{R}_{\mu \nu} \mathcal{R}^{\mu \nu}-\frac{(n-1)}{4(n-2)} \mathcal{R}^{2}\right)+\cdots\right],
\end{aligned}
$$

where $K=h^{\mu \nu} K_{\mu \nu}$ is the trace of the second fundamental form $K_{\mu \nu}=-\nabla_{(\mu} n_{\nu)}$, and $\mathcal{R}_{\mu \nu \rho \sigma}$ and its contractions denote curvatures in the boundary metric $h_{\mu \nu}=g_{\mu \nu}-n_{\mu} n_{\nu}$. The curvature-squared terms are needed for renormalisation only in dimensions $n>5$. The ellipses represent higher-order counterterms that would be needed in dimensions $n>7$. As 
mentioned above, we can side-step the need for the explicit forms of these higher counterterms for our simple spherically-symmetric static metrics.

In the scalar sector, we can have boundary and counterterms

$$
\begin{aligned}
\mathcal{L}_{\text {surf }}[\phi] & =\frac{\gamma}{16 \pi G} \sqrt{-h} n^{\mu} \phi \partial_{\mu} \phi, \\
\mathcal{L}_{\mathrm{ct}}[\phi] & =\frac{1}{16 \pi G} \sqrt{-h} \ell^{-1}\left(e_{1} \phi^{2}+e_{2} \phi^{3}+e_{3} \phi^{4}+\cdots\right) .
\end{aligned}
$$

The coefficients $e_{i}$ in the counterterms may be chosen in order to cancel further divergences in the holographic stress tensor that may arise. The constant $\gamma$ in the boundary term is typically a free parameter, which corresponds to the freedom to redefine the mass by means of a Legendre transformation that adds some function of the asymptotic scalar expansion coefficients. In certain cases where there is logarithmic $r$ dependence in the asymptotic expansions for the metric and scalar field, it is necessary to fix $\gamma$ in order to remove divergences in the holographic stress tensor.

Calculating the boundary stress tensor $T_{\alpha \beta}=(2 / \sqrt{-h}) \delta I / \delta h^{\alpha \beta}$, and substituting the form of our metric ansatz, we find that ${ }^{4}$ the renormalised holographic $T_{00}$ is obtained by taking the $r \longrightarrow \infty$ limit of

$$
\begin{array}{r}
T_{00}=\frac{1}{8 \pi G}\left[-\frac{(n-2) h \sqrt{f}}{r}+(n-2) h \ell^{-1} \sum_{p=0}^{[(n-2) / 2]} \frac{c_{p} \ell^{2 p}}{r^{2 p}}\right. \\
\left.-\gamma \sqrt{f} h \phi \phi^{\prime}+\frac{1}{2} \ell^{-1} h\left(e_{1} \phi^{2}+e_{2} \phi^{3}+\cdots\right)\right] .
\end{array}
$$

Here the constants $c_{p}$ are the universal ones we mentioned previously that are determined by requiring that there be no divergences in $T_{00}$ for the pure AdS case. This implies that

$$
c_{p}=\left(\begin{array}{c}
\frac{1}{2} \\
p
\end{array}\right)=\frac{\left(\frac{1}{2}\right) !}{p !\left(\frac{1}{2}-p\right) !},
$$

and so we can write

$$
\sum_{p=0}^{[(n-2) / 2]} \frac{c_{p} \ell^{2 p}}{r^{2 p}}=\frac{\ell \sqrt{f_{0}}}{r}-\sum_{p=[n / 2]}^{\infty} \frac{c_{p} \ell^{2 p}}{r^{2 p}}, \quad f_{0} \equiv r^{2} \ell^{-2}+1 .
$$

The holographic mass is obtained by integrating $T_{00}$ over the $(n-2)$ sphere at infinity, and hence

$$
M=\lim _{r \rightarrow \infty} r^{n-3} \ell \omega_{n-2} T_{00} .
$$

It therefore follows from (3.14) that the mass will be given by

$$
\begin{aligned}
M=\lim _{r \rightarrow \infty} \frac{r^{n-3} \omega_{n-2}}{8 \pi}[ & -\frac{(n-2)\left(\sqrt{f}-\sqrt{f_{0}}\right) h}{r} \\
& \left.-\gamma \sqrt{f} h \phi^{\prime} \phi+\frac{1}{2} \ell^{-1} h\left(e_{1} \phi^{2}+e_{2} \phi^{3}+\cdots\right)\right]+E_{\text {casimir }},
\end{aligned}
$$

\footnotetext{
${ }^{4}$ See section 3 of [23] for a detailed discussion of a closely related calculation for Einstein-Proca black holes in arbitrary dimensions.
} 
where the Casimir energy $E_{\text {casimir }}$ arises as an extra contribution from the leading $p=[n / 2]$ term in the sum on the right-hand side in (3.14) in the case that $n$ is odd. The Casimir energy when $n$ is odd, $n=2 q+1$, is given by

$$
E_{\text {casimir }}=-\frac{(2 q-1) \omega_{2 q-1} \ell^{2 q-2}}{8 \pi}\left(\begin{array}{c}
\frac{1}{2} \\
q
\end{array}\right) .
$$

Since our concern in the present work is just with the classical mass of the black holes, we shall drop the Casimir contribution from now on.

\subsection{AMD derivation of the mass}

Provided that a metric approaches AdS sufficiently rapidly, another convenient way of calculating the mass is by using the conformal method developed by Ashtekar, Magnon and Das $[16,17]$. This method, sometimes referred to as the AMD procedure, involves making a conformal rescaling of the metric to $\bar{g}_{\mu \nu}=\Omega^{2} g_{\mu \nu}$ with $\Omega \rightarrow 0, \bar{n}_{\mu}=\partial_{\mu} \Omega$ on the boundary, with $\bar{n}^{\mu}$ being a spacelike unit vector orthogonal to the spatial boundary. The AMD mass is then given by evaluating the integral

$$
M_{\mathrm{AMD}}=\frac{\ell}{8 \pi(n-3)} \int_{S^{n-2}} \overline{\mathcal{E}}^{\mu}{ }_{\nu} \xi^{\nu} d \bar{\Sigma}_{\mu}
$$

on the conformal boundary, where $\xi=\partial / \partial t$,

$$
\overline{\mathcal{E}}^{\mu}{ }_{\nu}=\bar{n}^{\rho} \bar{n}^{\sigma} \bar{C}^{\mu}{ }_{\rho \nu \sigma}
$$

and $\bar{C}_{\rho \nu \sigma}^{\mu}$ is the Weyl tensor of the conformally-rescaled metric. In the case of static metrics of the form (1.2), this amounts to evaluating

$$
M_{\mathrm{AMD}}=\left.\frac{\omega_{n-2}}{8 \pi(n-3)} r^{n-1} C^{0}{ }_{101}\right|_{r=\infty},
$$

where $C^{0}{ }_{101}$ is the $\operatorname{trtr}$ vielbein component of the Weyl tensor of the metric (1.2), which is given by

$$
C^{0}{ }_{101}=-\frac{(n-3)}{(n-1)}\left[\frac{f h^{\prime \prime}}{2 h}-\frac{f h^{\prime 2}}{4 h^{2}}+\frac{f^{\prime} h^{\prime}}{4 h}-\frac{f h^{\prime}}{2 r h}-\frac{f^{\prime}}{2 r}-\frac{1-f^{2}}{r^{2}}\right] .
$$

(A detailed discussion of the relation between the holographic calculation and the AMD calculation of the mass for Einstine-Scalar black holes appeared recently in [18].)

\section{Asymptotics and thermodynamics for $0<\sigma<1$}

Since the black-hole metric will be asymptotic to AdS at large distances, it follows that asymptotically the scalar field can include terms with the leading-order behaviours implied by (2.6), namely

$$
\phi(r) \sim \frac{\phi_{1}}{r^{(n-1-\sigma) / 2}}+\frac{\phi_{2}}{r^{(n-1+\sigma) / 2}}+\cdots,
$$


where $\phi_{1}$ and $\phi_{2}$ are constants. In the full non-linear theory, the scalar field will back-react on the metric, and in turn the metric will back-react on the scalar field. The asymptotic form of the full solutions can be found by making appropriate large- $r$ expansions for the functions $\phi(r), h(r)$ and $f(r)$, substituting them into the equations of motion (2.10), and solving for the coefficients in the expansions up to any desired order. For a general value of the mass $m$ of the scalar field it is quite complicated to set up the appropriate expansions, because the orders at which the back-reaction terms arise will interlace with the orders at which the original scalar field terms displayed in (4.1), and their descendants, will occur.

A relatively simple sub-class to consider is when the mass of the scalar field lies in the range corresponding to $0<\sigma<1$ (see (2.7)), namely

$$
-\frac{1}{4} \ell^{-2}(n-1)^{2}<m^{2}<-\frac{1}{4} \ell^{-2}\left[(n-1)^{2}-1\right]
$$

(Note that $\sigma=1$ corresponds to a conformally massless scalar, and it can arise in gauged supergravities in four and six dimensions, but not in five and seven dimensions. The black hole thermodynamics for $\sigma=1$ was obtained in [10].) In this range, limited at the lower end by the Breitenlohner-Freedman bound, the leading-order terms in the large- $r$ expansions will take the form

$$
\begin{aligned}
\phi & =\frac{\phi_{1}}{r^{(n-1-\sigma) / 2}}+\frac{\phi_{2}}{r^{(n-1+\sigma) / 2}}+\cdots, \\
h & =g^{2} r^{2}+1+\frac{\alpha}{r^{n-3}}+\cdots, \\
f & =g^{2} r^{2}+1+\frac{b}{r^{n-3-\sigma}}+\frac{\beta}{r^{n-3}}+\cdots .
\end{aligned}
$$

Note that the Schwarzschild-AdS black hole corresponds to

$$
\alpha=\beta, \quad \phi_{1}=\phi_{2}=b=0,
$$

with all higher-order terms vanishing as well.

Substituting the expansions (4.3) into the equations of motion and solving for the first few coefficients, we find that

$$
b=\frac{(n-1-\sigma) \phi_{1}^{2}}{4(n-2) \ell^{2}}, \quad \beta=\alpha+\frac{\left[(n-1)^{2}-\sigma^{2}\right] \phi_{1} \phi_{2}}{2(n-1)(n-2) \ell^{2}} .
$$

The expression (3.5) for $\delta \mathcal{H}$, evaluated at infinity, then gives

$$
\delta \mathcal{H}_{\infty}=\frac{\omega_{n-2}}{16 \pi}\left[-(n-2) \delta \alpha+\frac{\sigma}{2(n-1) \ell^{2}}\left[(n-1+\sigma) \phi_{2} \delta \phi_{1}-(n-1-\sigma) \phi_{1} \delta \phi_{2}\right]\right] .
$$

(Each of the two terms in (3.5) has a divergence of order $r^{\sigma}$ alt large $r$, but these cancel when the two terms are added.) Note that (4.6) is precisely of the form (1.6), with $E$ in this case given by $(1.8)$ with $K\left(\phi_{1}, \phi_{2}\right)=0$.

It is straightforward to evaluate the expression (3.16) for the holographic mass for these generic Einstein-Scalar black holes with $0<\sigma<1$. We find that in order to remove a divergence at order $r^{\sigma}$, the counterterm coefficient $e_{1}$ must be chosen such that

$$
e_{1}=\frac{1}{8}(n-1-\sigma)(1-4 \gamma) \text {. }
$$


The higher-order counterterms in (3.11) are not needed for removing divergences in these examples, and so we can simply set $e_{i}=0$ for $i \geq 2$. The holographic mass is then given by

$$
M=\frac{\omega_{n-2}}{16 \pi}\left[-(n-2) \alpha+\frac{[(n-1)(4 \gamma-1)+\sigma] \sigma}{2(n-1) \ell^{2}} \phi_{1} \phi_{2}\right] .
$$

The most natural choice for the free parameter $\gamma$ is to choose it so that the mass is simply proportional to $\alpha$, i.e. proportional to the coefficient of $r^{3-n}$ in the metric function $g_{00}$. In view of (4.7), this is achieved by taking

$$
\gamma=\frac{n-1-\sigma}{4(n-1)}, \quad e_{1}=\frac{(n-1-\sigma) \sigma}{8(n-1)} .
$$

This then implies that the holographic mass of the Einstein-Scalar black holes with $0<$ $\sigma<1$ is simply given by ${ }^{5}$

$$
M=-\frac{(n-2) \omega_{n-2} \alpha}{16 \pi} .
$$

Another reason for favouring the choice of parameters (4.9) that leads to the mass (4.10) is that we obtain exactly the same result for the mass if we use the AMD conformal procedure, which is given by (3.20) and (3.21) for our metrics.

The evaluation of $\delta \mathcal{H}$ on the outer horizon gives $T \delta S$, and so from (1.4) and we obtain the first law of thermodynamics

$$
d M=T d S-\frac{\sigma \omega_{n-2}}{32 \pi(n-1) \ell^{2}}\left[(n-1+\sigma) \phi_{2} d \phi_{1}-(n-1-\sigma) \phi_{1} d \phi_{2}\right] .
$$

Although (4.11) was derived for $\sigma$ lying in the restricted range $0<\sigma<1$, it also reproduces the result obtained in [10] if we set $\sigma=1$. Note that since $\phi_{1}$ and $\phi_{2}$ have the dimensions of (Length) $)^{(n-1 \mp \sigma) / 2}$ respectively, the ratio $\phi_{1}^{n-1-\sigma} / c \phi_{2}^{n-1+\sigma}$ is dimensionless. The scalar contribution to the first law would therefore vanish if this ratio were equal to a fixed dimensionless number, which could include 0 or $\infty$.

\section{Further examples outside $0<\sigma<1$}

The general discussion in the previous section was for $n$-dimensional Einstein-Scalar black holes with $0<\sigma<1$. This corresponds to the range of negative mass-squared values for the scalar field given in (4.2), with $\sigma=0$ corresponding to the Breitenlohner-Freedman limit. For values of the scalar mass that lie outside this range, one really has to consider examples on a case by case basis, since the structure of the dominant terms in the asymptotic expansions become rather dependent on the range for the scalar mass. Below, we shall present a few illustrative examples.

First, we note that there is a natural upper limit to the mass-squared range we should consider, namely $m^{2}=0$. This can be seen by noting, as is already evident in (4.3), that the dominant back-reaction of the scalar field on the metric at large $r$ occurs in the function

\footnotetext{
${ }^{5}$ Other choices for the parameter $\gamma$ would amount to adding a constant multiple of $\phi_{1} \phi_{2}$ to the definition of the mass. This would be rather like the Legendre transformations that one makes between different forms of energy in standard thermodynamics, such as the free energy, Helmholtz energy, enthalpy, and so on.
} 
$f$ at order $1 / r^{n-3-\sigma}$. If this power were actually to exceed $r^{2}$ then the back-reacted metric would no longer be asymptotic to AdS. This implies we must have

$$
\sigma<n-1, \text { and hence } m^{2}<0 \text {. }
$$

We now proceed to consider some specific examples. These serve to illustrate some of the new features that can arise in certain cases.

\section{$5.1 \quad$ Special examples}

\subsection{1 $n=4, \sigma=1$}

The value $\sigma=1$ corresponds to the cases that arise for gauged supergravity theories in four dimensions. In fact, these potentials are typically even functions of $\phi$, and so $\gamma_{3}=\gamma_{5}=\cdots=0$. We shall, however, keep $\gamma_{3}, \gamma_{5}$, etc., arbitrary and non-zero for now, since this allows greater generality in our results. It also introduces new features in the solutions.

We find that the scalar and metric functions have large- $r$ expansions of the form

$$
\begin{aligned}
\phi & =\frac{\phi_{1}}{r}+\frac{\phi_{2}}{r^{2}}-\frac{3 \gamma_{3} \ell^{2} \phi_{1}^{2} \log r}{r^{2}}+\cdots, \\
h & =r^{2} \ell^{-2}+1+\frac{\alpha}{r}+\cdots, \\
f & =r^{2} \ell^{-2}+1+\frac{1}{4} \ell^{-2} \phi_{1}^{2}+\frac{f_{1}}{r}-\frac{2 \gamma_{3} \phi_{1}^{3} \log r}{r}+\cdots,
\end{aligned}
$$

with

$$
f_{1}=\alpha+\frac{1}{3} \gamma_{3} \phi_{1}^{3}+\frac{2}{3} \ell^{-2} \phi_{1} \phi_{2} .
$$

Note that the terms with $\log r$ dependence are associated with having a non-vanishing coefficient $\gamma_{3}$ in the Taylor expansion of the scalar potential. This feature also persists at higher orders in the expansion.

The Wald formula (3.5) gives

$$
\delta \mathcal{H}_{\infty}=\frac{\omega_{2}}{16 \pi}\left[-2 \delta \alpha+\gamma_{3} \phi_{1}^{2} \delta \phi_{1}+\frac{1}{3} \ell^{-2}\left(2 \phi_{2} \delta \phi_{1}-\phi_{1} \delta \phi_{2}\right)\right] .
$$

The AMD calculation of the mass, using (3.20), gives

$$
M_{\mathrm{AMD}}=-\frac{\alpha \omega_{2}}{8 \pi} .
$$

If $\gamma_{3} \neq 0$, we find in the calculation (3.16) of the holographic mass that it is necessary to take $e_{1}=\frac{1}{2}-2 \gamma$ in order to remove a linear divergence, and also to set $\gamma=\frac{1}{6}$ to remove a logarithmic divergence. The result for the holographic mass is then

$$
M_{\mathrm{hol}}=\frac{\omega_{2}}{16 \pi}\left[-2 \alpha+\frac{1}{3} \gamma_{3} \phi_{1}^{3}\right],
$$

and so we find

$$
\delta \mathcal{H}_{\infty}=\delta M_{\mathrm{hol}}+\frac{\omega_{2}}{48 \pi \ell^{2}}\left(2 \phi_{2} \delta \phi_{1}-\phi_{1} \delta \phi_{2}\right) .
$$

Note that $M_{\text {hol }} \neq M_{\mathrm{AMD}}$ in this $\gamma_{3} \neq 0$ case. 
If we were to take $\gamma_{3}=0$, then the absence of the $\log r$ terms in the asymptotic expansion means that there is now only a single, linear, divergence in the calculation of the holographic mass, and so only the counterterm coefficient $e_{1}$ is determined, $e_{1}=\frac{1}{2}-2 \gamma$, leaving $\gamma$ arbitrary. The holographic mass is now given by

$$
M_{\mathrm{hol}}=\frac{\omega_{2}}{16 \pi}\left[-2 \alpha-\frac{1}{3} \ell^{-2}(1-6 \gamma) \phi_{1} \phi_{2}\right] .
$$

We could make the simple choice $\gamma=\frac{1}{6}$, just as was forced in the $\gamma_{3} \neq 0$ case, and then the holographic mass agrees with the AMD mass (5.5). We then obtain the same Wald variational result (5.7) as in the $\gamma_{3} \neq 0$ case.

\subsection{2 $n=5, \sigma=1$}

This example, for which we have $\hat{m}^{2}=-\frac{15}{4} \ell^{-2}$, lies at the upper limit of the range $0<\sigma<1$ that we discussed above. In fact, its leading-order terms fit within the general pattern of the $0<\sigma<1$ solutions discussed previously, but we have included it here because by considering a specific example we can easily illustrate the pattern of some of the higher-order terms in the asymptotic expansion. Thus we find

$$
\begin{aligned}
\phi & =\frac{\phi_{1}}{r^{\frac{3}{2}}}+\frac{\phi_{2}}{r^{\frac{5}{2}}}+\frac{4 \ell^{2} \gamma_{3} \phi_{1}^{2}}{r^{3}}+\frac{3 \ell^{2} \phi_{1}}{8 r^{\frac{7}{2}}}+\cdots, \\
h & =\ell^{-2} r^{2}+1+\frac{\alpha}{r^{2}}+\frac{\phi_{1}^{2}}{20 r^{3}}+\cdots, \\
f & =\ell^{-2} r^{2}+1+\frac{\phi_{1}^{2}}{4 \ell^{2} r}+\frac{\beta}{r^{2}}+\frac{8 \gamma_{3} \phi_{1}^{3}}{3 r^{\frac{5}{2}}}+\frac{27 \ell^{2} \phi_{1}^{2}+20 \phi_{2}^{2}}{48 \ell^{2} r^{3}}+\cdots .
\end{aligned}
$$

Here we are seeing a term at order $r^{-5 / 2}$ in the metric function $f$. This is a consequence of considering a general scalar potential that has odd as well as even powers of $\phi$, implying that there will be back-reaction terms from the scalar field in the metric functions with half-integer as well as integer powers of $1 / r$. (Note that the $r^{-5 / 2}$ term in $f$ vanishes if $\gamma_{3}=0$.)

The Wald calculation of $\delta \mathcal{H}$ in (3.5) gives

$$
\delta \mathcal{H}_{\infty}=\frac{\omega_{3}}{16 \pi}\left[-3 \delta \alpha+\frac{1}{8} \ell^{-2}\left(5 \phi_{2} \delta \phi_{1}-3 \phi_{1} \delta \phi_{2}\right)\right] .
$$

The AMD formula (3.20) for the mass of the black hole gives

$$
M_{\mathrm{AMD}}=-\frac{3 \omega_{3} \alpha}{16 \pi} .
$$

On the other hand, the holographic mass calculated from (3.16) turns out to be

$$
M_{\mathrm{hol}}=\frac{\omega_{3}}{16 \pi}\left[-3 \alpha+\frac{1}{8} \ell^{-2}(16 \gamma-3) \phi_{1} \phi_{2}\right],
$$

with the counterterm $e_{1}$ determined to be $e_{1}=\frac{3}{4}(1-4 \gamma)$ in order to remove a divergence. The freedom to choose the boundary coefficient $\gamma$ represents an arbitrariness associated with making a Legendre transform to a different energy function. It would be natural in this case to take $\gamma=\frac{3}{16}$, so that the holographic mass would coincide with the AMD mass. 


\subsection{3 $n=5, \sigma=2$}

In this case, we have $\hat{m}^{2}=-3 \ell^{-2}$. Now we have

$$
\begin{aligned}
& \phi=\frac{\phi_{1}}{r}-\frac{3 \ell^{2} \gamma_{3} \phi_{1}^{2}}{r^{2}}+\frac{\phi_{2}+c \log r}{r^{3}}+\cdots, \\
& h=\ell^{-2} r^{2}+1+\frac{\alpha+a \log r}{r^{2}}+\cdots, \\
& f=\ell^{-2} r^{2}+1+\frac{1}{6} \ell^{-2} \phi_{1}^{2}-\frac{4 \gamma_{3} \phi_{1}^{3}}{3 r}+\frac{\beta+b \log r}{r^{2}}+\cdots,
\end{aligned}
$$

where

$$
\begin{aligned}
& a=-\frac{1}{12} \phi_{1}^{2}, \quad b=-\frac{1}{3} \phi_{1}^{2}+\left(\frac{9}{2} \ell^{2} \gamma_{3}^{2}-\gamma_{4}-\frac{1}{12} \ell^{-2}\right) \phi_{1}^{4}, \\
& c=-\frac{1}{2} \ell^{2} \phi_{1}+\left(9 \ell^{4} \gamma_{3}^{2}-2 \ell^{2} \gamma_{4}-\frac{1}{6}\right) \phi_{1}^{3}, \\
& \beta=\alpha+\frac{1}{2} \ell^{-2} \phi_{1} \phi_{2}+\frac{3}{16} \phi_{1}^{2}+\frac{1}{48} \ell^{-2}\left(1+4 \ell^{2} \gamma_{4}+126 \ell^{4} \gamma_{3}^{2}\right) \phi_{1}^{4} .
\end{aligned}
$$

The Wald formula (3.5) gives

$$
\begin{aligned}
\delta \mathcal{H}_{\infty} & =\frac{\omega_{3}}{16 \pi}\left[-3 \delta \alpha+\frac{1}{2} \ell^{-2}\left(3 \phi_{2} \delta \phi_{1}-\phi_{1} \delta \phi_{2}\right)+\delta K\right], \\
K & =\frac{3}{16} \phi_{1}^{2}+\frac{1}{4}\left(\gamma_{4}+\frac{1}{12} \ell^{-2}-\frac{9}{2} \ell^{2} \gamma_{3}^{2}\right) \phi_{1}^{4} .
\end{aligned}
$$

(Note that the two terms in (3.5) each separately have divergences at large $r$, but the sum is finite.)

The AMD mass formula (3.20) has a logarithmic divergence at large $r$, with a coefficient proportional to $\phi_{1}^{2}$. The holographic mass, given by (3.16) is also in general logarithmically divergent, but we can obtain a finite answer if we restrict the coefficients $\gamma_{3}$ and $\gamma_{4}$ in the scalar potential so that

$$
\gamma_{4}=-\frac{1}{12} \ell^{-2}+\frac{9}{2} \ell^{2} \gamma_{3}^{2}
$$

In addition, we must choose the coefficients of the boundary term and counterterms for the scalar field so that

$$
\gamma=\frac{1}{4}, \quad e_{1}=0, \quad e_{2}=\frac{1}{2} \ell^{2} \gamma_{3} .
$$

The condition (5.16) on $\gamma_{4}$ that is needed in order to obtain a finite holographic mass is precisely such that it removes the $\phi_{1}^{4}$ term in $K$ in (5.15). The $\phi^{4}$ counterterm, with coefficient $e_{3}$, is not needed in order to remove divergences from the holographic mass. However, it does make a finite contribution to the mass that is proportional to $\phi_{1}^{4}$, and by choosing it to have the value

$$
e_{3}=-\frac{1}{48}+\frac{9}{2} \ell^{4} \gamma_{3}^{2}
$$

one can remove all $\phi_{1}^{4}$ contributions to $M_{\text {hol }}$, leaving just

$$
M_{\mathrm{hol}}=\frac{\omega_{3}}{16 \pi}\left[-3 \alpha+\frac{3}{16} \phi_{1}^{2}+\frac{1}{2} \ell^{-2} \phi_{1} \phi_{2}\right] .
$$


We then find from (5.15) that

$$
\delta \mathcal{H}_{\infty}=\delta M_{\mathrm{hol}}+\frac{\omega_{3}}{16 \pi \ell^{2}}\left(\phi_{2} \delta \phi_{1}-\phi_{1} \delta \phi_{2}\right) .
$$

\subsection{4 $n=5, \sigma=\frac{5}{2}$}

In this case, we have $\hat{m}^{2}=-\frac{39}{16} \ell^{-2}$. We have

$$
\begin{aligned}
\phi & =\frac{\phi_{1}}{r^{\frac{3}{4}}}+\frac{c_{1}}{r^{\frac{3}{2}}}+\frac{c_{2}}{r^{\frac{9}{4}}}+\frac{c_{3}}{r^{\frac{11}{4}}}+\frac{c_{4}}{r^{\frac{12}{4}}}+\frac{\phi_{2}}{r^{\frac{13}{4}}}+\cdots, \\
h & =\ell^{-2} r^{2}+1+\frac{a_{1}}{r^{\frac{3}{2}}}+\frac{\alpha}{r^{2}}+\cdots, \\
f & =g^{2} r^{2}+b_{1} r^{\frac{1}{2}}+1+\frac{b_{2}}{r^{\frac{1}{4}}}+\frac{b_{3}}{r}+\frac{b_{4}}{r^{\frac{3}{2}}}+\frac{b_{5}}{r^{\frac{7}{4}}}+\frac{\beta}{r^{2}}+\cdots,
\end{aligned}
$$

where

$$
\begin{array}{rlrl}
a_{1} & =-\frac{5}{28} \phi_{1}^{2}, & b_{1} & =\frac{1}{8} \ell^{-2} \phi_{1}^{2}, \quad b_{2}=-\frac{16}{21} \gamma_{3} \phi_{1}^{3}, \\
b_{3} & =\frac{1}{12544} \ell^{-2}\left(-637-12544 \ell^{2} \gamma_{4}+59392 \ell^{4} \gamma_{3}^{2}\right) \phi_{1}^{4}, \\
b_{4} & =-\frac{27}{64} \phi_{1}^{2}, & b_{5} & =\frac{1}{882}\left(1925 \gamma_{3}-3136 \gamma_{5}+30464 \ell^{2} \gamma_{3} \gamma_{4}-55296 \ell^{4} \gamma_{3}^{3}\right) \phi_{1}^{5}, \\
c_{1} & =-\frac{16}{7} \ell^{2} \gamma_{3} \phi_{1}^{2}, & c_{2} & =\frac{1}{672}\left(-105-1792 \ell^{2} \gamma_{4}+6144 \ell^{4} \gamma_{3}^{2}\right) \phi^{3}, \\
c_{3} & =-\frac{15}{16} \ell^{2} \phi_{1}, & c_{4} & =\frac{\ell^{2}}{441}\left(2275 \gamma_{3}-3920 \gamma_{5}+34048 \ell^{2} \gamma_{3} \gamma_{4}-55296 \ell^{4} \gamma_{3}^{3}\right) \phi_{1}^{4} .
\end{array}
$$

Each of the two terms in the Wald formula (3.5) contains divergences at large $r$ but the sum of the two gives the simple and finite result

$$
\delta \mathcal{H}_{\infty}=\frac{\omega_{3}}{16 \pi}\left[-3 \delta \alpha+\frac{5}{32} \ell^{-2}\left(13 \phi_{2} \delta \phi_{1}-3 \phi_{1} \delta \phi_{2}\right)\right] .
$$

In this example the rate at which the metric approaches AdS is too slow, if $\phi_{1} \neq 0$, for the AMD formula (3.20) to give a finite mass; there is a divergence of order $r^{1 / 2}$ with a coefficient proportional to $\phi_{1}^{2}$. On the other hand, the holographic mass formula (3.16) does give a finite result, although we must now add the extra counterterms with coefficients $e_{2}, e_{3}$ and $e_{4}$ in (3.11) in order to cancel divergences. Specifically, we find now that we must choose

$$
\begin{aligned}
\gamma & =\frac{1}{4}, \quad e_{1}=0, \quad e_{2}=\frac{2}{7} \ell^{2} \gamma_{3}, \quad e_{3}=\frac{3}{64}-\frac{72}{49} \ell^{4} \gamma_{3}^{2}+\ell^{2} \gamma_{4}, \\
e_{4} & =-\frac{39}{14} \ell^{2} \gamma_{3}+\frac{20736}{343} \ell^{6} \gamma_{3}^{3}-\frac{288}{7} \ell^{4} \gamma_{3} \gamma_{4}+6 \ell^{2} \gamma_{5},
\end{aligned}
$$

and then the holographic mass is given by

$$
M_{\mathrm{hol}}=\frac{\omega_{3}}{16 \pi}\left[-3 \alpha+\frac{25}{32} \ell^{-2} \phi_{1} \phi_{2}\right] .
$$

From (5.23) we therefore have

$$
\delta \mathcal{H}_{\infty}=\delta M_{\mathrm{hol}}+\frac{5 \omega_{3}}{64 \pi \ell^{2}}\left(\phi_{2} \delta \phi_{1}-\phi_{1} \delta \phi_{2}\right) .
$$




\subsection{5 $n=7, \sigma=2$}

This can arise in gauged supergravities in seven dimensions. We have

$$
\begin{aligned}
\phi= & \frac{\phi_{1}}{r^{2}}-\frac{\ell^{2} \phi_{1}\left(4+3 \gamma_{3} \phi_{1}\right) \log r}{2 r^{4}}+\frac{\phi_{2}}{r^{4}}+\cdots, \\
h= & \ell^{-2} r^{2}+1-\frac{2 \phi_{1}^{2} \log r}{15 r^{4}}+\frac{\alpha}{r^{4}}+\cdots, \\
f= & \ell^{-2} r^{2}+1+\frac{\phi_{1}^{2}}{5 \ell^{2} r^{2}}-\frac{2 \phi_{1}^{2}\left(3+2 \gamma_{3} \phi_{1}\right) \log r}{5 r^{4}} \\
& +\frac{\alpha}{r^{4}}+\frac{\phi_{1}\left(\ell^{2} \phi_{1}\left(13+3 \gamma_{3} \phi_{1}\right)+24 \phi_{2}\right)}{45 \ell^{2} r^{4}}+\cdots .
\end{aligned}
$$

The Wald formula (3.5) gives

$$
\delta \mathcal{H}_{\infty}=\frac{\omega_{5}}{16 \pi}\left[-5 \delta \alpha+\frac{2}{3} \ell^{-2}\left(2 \phi_{2} \delta \phi_{1}-\phi_{1} \delta \phi_{2}\right)+\delta K\right], \quad K=\frac{5}{9} \phi_{1}^{2}+\frac{1}{6} \gamma_{3} \phi_{1}^{3} .
$$

The AMD mass is divergent in this case, since the metric approaches AdS too slowly. In the calculation of the holographic mass, a quadratic divergence is removed by the $\phi^{2}$ counterterm in the standard way, provided that

$$
e_{1}=1-4 \gamma
$$

However, this leaves a logarithmically-divergent contribution to the mass, of the form

$$
M_{\log }=\frac{\omega_{5}}{16 \pi}\left[2(1-4 \gamma) \phi_{1}^{2}+(1-6 \gamma) \gamma_{3} \phi_{1}^{3}\right] \log r .
$$

The value of the boundary term coefficient $\gamma$ should not depend on the specific parameters (such as $\phi_{1}$ ) of the solution, and so this divergence seemingly cannot be removed in general, and there does not appear to be any additional local counterterm that could do the job. One possible resolution is to add a counterterm proportional to $k \sqrt{-h} \phi^{3} \log \left(\frac{1}{20} \ell^{2} \mathcal{R}\right)$, which will give an additional logarithmically-divergent contribution

$$
M_{\log , \text { extra }}=\frac{\omega_{5} k}{\pi} \phi_{1}^{3} \log r .
$$

Choosing $\gamma=\frac{1}{4}$ and $k=\frac{1}{32} \gamma_{3}$, the divergences are now cancelled, and the mass becomes

$$
M=\frac{\omega_{5}}{16 \pi}\left[-5 \alpha+\frac{5}{9} \phi_{1}^{2}+\left(\frac{5}{12} \gamma_{3}+\ell^{-2} e_{2}\right) \phi_{1}^{3}+\frac{1}{3} \ell^{-2} \phi_{1} \phi_{2}\right] .
$$

A convenient choice for the counterterm coefficient $e_{2}$ (which is not needed for removing any divergence) is to take $e_{2}=-\frac{1}{4} \ell^{2} \gamma_{3}$, leading to $M=\omega_{5} /(16 \pi)\left(-5 \alpha+K+\frac{1}{3} \ell^{-2} \phi_{1} \phi_{2}\right)$, where $K$ is defined in (5.28). The first law then becomes

$$
d M=T d S-\frac{\omega_{5}}{16 \pi \ell^{2}}\left(\phi_{2} d \phi_{1}-\phi_{1} d \phi_{2}\right) .
$$

This example provides an illustration of the fact that the calculation of the holographic mass can become problematical in certain circumstances, especially when there is logarithmic dependence on the radial coordinate in the asymptotic expansions of the scalar and 
metric functions. An alternative way to define the mass in this example, which sidesteps the possibly questionable introduction of a non-local counterterm in the holographic renormalisation, is simply to use the Wald calculation of the first law itself as a way of determining the mass. As we discussed in the introduction, we can read off the energy $E$, modulo the freedom to make a Legendre transformation that adds a constant multiple of $\phi_{1} \phi_{2}$, by writing $\delta \mathcal{H}_{\infty}$ as the sum of $\delta E$ plus the non-integrable contribution involving the $\phi_{2} \delta \phi_{1}$ and $\phi_{1} \delta \phi_{2}$ terms. If we choose to fix this redefinition ambiguity by writing the ratio of the two terms in the non-integrable piece as in (5.33), then the mass will be precisely the one given in (5.32).

\subsubsection{The Breitenlohner-Freedman limit $\sigma=0$}

This is the case where $m^{2}$ saturates the Breitenlohner-Freedman bound, and hence $\sigma=0$. This can arise in five-dimensional gauged supergravities. The large- $r$ expansion of the scalar and metric fields takes the form

$$
\begin{aligned}
& \phi=\frac{\phi_{1} \log r+\phi_{2}}{r^{(n-1) / 2}}+\cdots, \quad h=\ell^{-2} r^{2}+1+\frac{\alpha}{r^{n-3}}+\cdots, \\
& f=\ell^{-2} r^{2}+1+\frac{b_{1}(\log r)^{2}+b_{2} \log r+\beta}{r^{n-3}}+\cdots,
\end{aligned}
$$

where

$$
\begin{aligned}
b_{1} & =\frac{(n-1) \phi_{1}^{2}}{4(n-2) \ell^{2}}, \quad b_{2}=\frac{\phi_{1}\left((n-1) \phi_{2}-\phi_{1}\right)}{2(n-2) \ell^{2}}, \\
\beta & =\alpha+\frac{(n-1)^{2} \phi_{2}^{2}-2(n-1) \phi_{1} \phi_{2}+2 \phi_{1}^{2}}{4(n-1)(n-2) \ell^{2}} .
\end{aligned}
$$

The Wald formula gives

$$
\delta \mathcal{H}_{\infty}=\frac{\omega_{n-2}}{16 \pi}\left[-(n-2) \delta \alpha+\frac{1}{2} \ell^{-2}\left(\phi_{2} \delta \phi_{1}-\phi_{1} \delta \phi_{2}\right)-\frac{\phi_{1} \delta \phi_{1}}{(n-1) \ell^{2}}\right] .
$$

The AMD formula (3.20) gives the mass

$$
M_{\mathrm{AMD}}=-\frac{(n-2) \omega_{n-2} \alpha}{16 \pi} .
$$

Using instead the expression (3.16) for the holographic mass, we find that in order to remove logarithmic divergences we must take

$$
e_{1}=0, \quad \gamma=\frac{1}{4},
$$

and this then implies

$$
M_{\mathrm{hol}}=\frac{\omega_{n-2}}{16 \pi}\left[-(n-2) \alpha-\frac{\phi_{1}^{2}}{2(n-1) \ell^{2}}\right] .
$$

Thus in terms of the holographic mass, we find

$$
\delta \mathcal{H}_{\infty}=\delta M_{\mathrm{hol}}+\frac{\omega_{n-2}}{32 \pi \ell^{2}}\left(\phi_{2} \delta \phi_{1}-\phi_{1} \delta \phi_{2}\right) .
$$




\subsubsection{Imaginary $\sigma$}

This corresponds to the situation where $m^{2}$ is more negative than the BreitenlohnerFreedman bound. Within the framework of a purely static ansatz for the fields, one still obtains perfectly regular Einstein-Scalar black holes. However, since the scalar field is now genuinely tachyonic, with complex energy eigenstates, one would find exponentiallygrowing time-dependent instabilities of the static solutions. For our present purposes, it is still of interest to consider the static solutions in their own right, since such instabilities will not be present within the framework of purely static solutions.

Taking

$$
\sigma=\mathrm{i} \tilde{\sigma}
$$

we find that the leading-order terms in a large-r expansion of the scalar and metric functions take the form

$$
\begin{aligned}
& \phi=\frac{\phi_{1} \cos \left(\frac{1}{2} \tilde{\sigma} \log r\right)+\phi_{2} \sin \left(\frac{1}{2} \tilde{\sigma} \log r\right)}{r^{(n-1) / 2}}+\cdots, \\
& h=\ell^{-2} r^{2}+1+\frac{\alpha}{r^{n-3}}+\cdots, \\
& f=\ell^{-2} r^{2}+1+\frac{b_{1} \cos (\tilde{\sigma} \log r)+b_{2} \sin (\tilde{\sigma} \log r)+\beta}{r^{n-3}}+\cdots,
\end{aligned}
$$

with

$$
\begin{aligned}
b_{1} & =\frac{1}{8(n-2) \ell^{2}}\left[(n-1)\left(\phi_{1}^{2}-\phi_{2}^{2}\right)-2 \tilde{\sigma} \phi_{1} \phi_{2}\right], \\
b_{2} & =\frac{1}{8(n-2) \ell^{2}}\left[\tilde{\sigma}\left(\phi_{1}^{2}-\phi_{2}^{2}\right)+2(n-1) \phi_{1} \phi_{2}\right], \\
\beta & =\alpha+\frac{\left(\phi_{1}^{2}+\phi_{2}^{2}\right)\left[(n-1)^{2}+\tilde{\sigma}^{2}\right]}{8(n-1)(n-2) \ell^{2}} .
\end{aligned}
$$

The Wald formula gives

$$
\begin{aligned}
\delta \mathcal{H}_{\infty} & =\frac{\omega_{n-2}}{16 \pi}\left[-(n-2) \delta \alpha-\frac{1}{4} \ell^{-2} \tilde{\sigma}\left(\phi_{2} \delta \phi_{1}-\phi_{1} \delta \phi_{2}\right)+\delta K\right], \\
K & =-\frac{\tilde{\sigma}^{2}}{8(n-1) \ell^{2}}\left(\phi_{1}^{2}+\phi_{2}^{2}\right) .
\end{aligned}
$$

Calculating the mass using the AMD formula (3.20), we find simply

$$
M_{\mathrm{AMD}}=-\frac{(n-2) \omega_{n-2} \alpha}{16 \pi} .
$$

Calculating the holographic mass from (3.16), we find that in order to remove dependence of the large- $r$ expansion on the trigonometric functions of $\log r$ we must choose

$$
e_{1}=0, \quad \gamma=\frac{1}{4},
$$

then leading to

$$
M_{\mathrm{hol}}=\frac{\omega_{n-2}}{16 \pi}\left[-(n-2) \alpha-\frac{\tilde{\sigma}^{2}}{8(n-1) \ell^{2}}\left(\phi_{1}^{2}+\phi_{2}^{2}\right)\right] .
$$


Thus from (5.44) we find

$$
\delta \mathcal{H}_{\infty}=\delta M_{\mathrm{hol}}-\frac{\tilde{\sigma} \omega_{n-2}}{64 \pi \ell^{2}}\left(\phi_{2} \delta \phi_{1}-\phi_{1} \delta \phi_{2}\right) .
$$

\section{Numerical analysis of black holes in gauged supergravities}

Recently, there has been progress in constructing exact black hole solutions in Einstein gravity coupled to scalar fields. The construction treats the scalar potential as a specifiable function that is to be determined by the third equation of (2.10). Making an ansatz that relates the metric functions and the scalar field, one can then obtain exact solutions [2530]. However, in all these constructions the solutions are not generic, and one has either $\phi_{1}=0$ or $\phi_{2}=0$, so the first law of thermodynamics is therefore unmodified by the scalar charges. Thus these solutions do not provide examples for illustrating the first law we have obtained in this paper.

In this section, we use numerical methods to construct solitons and black holes in which both $\phi_{1}$ and $\phi_{2}$ are non-vanishing. Furthermore, we focus on such solutions in gauged supergravities. A class of scalar potentials in gauged supergravities in $n$ dimensions can be summarised in terms of the following superpotential [31]:

$$
W=\frac{N g(n-3)}{\sqrt{2}}\left(e^{-\frac{1}{2} a_{1} \phi}-\frac{a_{1}}{a_{2}} e^{-\frac{1}{2} a_{2} \phi}\right)
$$

where

$$
a_{1}^{2}=\frac{4}{N}-\frac{2(n-3)}{n-2}, \quad a_{1} a_{2}=-\frac{2(n-3)}{n-2} .
$$

The scalar potential is then given by

$$
V=\left(\frac{d W}{d \phi}\right)^{2}-\frac{n-1}{2(n-2)} W^{2}
$$

Thus we have

$$
\begin{aligned}
& N=1: \quad V=-(n-1) g^{2}\left[(n-3) e^{-\frac{\sqrt{2} \phi}{\sqrt{(n-1)(n-2)}}}+e^{\left.\frac{\sqrt{2}(n-3) \phi}{\sqrt{(n-1)(n-2)}}\right]},\right. \\
& N=2: \quad V=-g^{2}\left[(n-3)^{2} e^{-\frac{\sqrt{2} \phi}{\sqrt{n-2}}}+4(n-3) e^{\frac{(n-4) \phi}{\sqrt{2(n-2)}}}-(n-5) e^{\frac{\sqrt{2}(n-3) \phi}{\sqrt{n-2}}}\right] .
\end{aligned}
$$

Both of these scalar potentials can be embedded in appropriate gauged supergravities in $n=4,5,6$ and 7 dimensions. The $N=1$ and $N=2$ potentials were summarised first in [32] and [33] respectively. At the linearised level, we have

$$
V=-(n-1)(n-2) g^{2}-(n-3) g^{2} \phi^{2}+\cdots .
$$

This implies that $m^{2}=-2(n-3) g^{2}$ and hence $\sigma^{2}=(n-5)^{2}$. The asymptotic behaviour for various values of $\sigma$ in general dimensions was discussed in the previous sections. 


\subsection{Inner expansions}

In order to perform numerical calculations, we start with a solution in an inner region, and then integrate it out to infinity. As discussed earlier, for $\sigma$ in an appropriate range, this procedure cannot fail to give a solution with good behaviour near infinity, since both solutions to the relevant second-order equations have good asymptotic behaviour. There are two classes of solution that are of interest. One is of the solitonic type, where $r$ starts at $r=0$. Regularity at $r=0$ requires that $h(r)$ and $\phi(r)$ approach constants, and $f(r)$ approaches 1, as $r$ goes to zero. Performing Taylor expansions, we find

$$
\begin{aligned}
& h=h_{0}\left(1-\frac{V\left(\phi_{0}\right)}{(n-1)(n-2)} r^{2}-\frac{V^{\prime}\left(\phi_{0}\right)^{2}}{4\left(n^{2}-1\right)(n-2)} r^{4}+\cdots\right), \\
& f=1-\frac{V\left(\phi_{0}\right)}{(n-1)(n-2)} r^{2}-\frac{n V^{\prime}\left(\phi_{0}\right)^{2}}{2\left(n^{2}-1\right)(n-1)(n-2)} r^{4}+\cdots, \\
& \phi=\phi_{0}+\frac{V^{\prime}\left(\phi_{0}\right)}{2(n-1)} r^{2}+\cdots .
\end{aligned}
$$

The other class of solutions of interest are black holes. Assuming that the horizon is located at $r=r_{0}>0$, we find that the near-horizon expansion is given by

$$
\begin{aligned}
& h=h_{1}\left[\left(r-r_{0}\right)+h_{2}\left(r-r_{0}\right)^{2}+\cdots\right], \\
& f=f_{1}\left(r-r_{0}\right)+f_{2}\left(r-r_{0}\right)^{2}+\cdots, \\
& \phi=\phi_{0}+\frac{(n-2) r_{0} V^{\prime}\left(\phi_{0}\right)}{(n-2)(n-3)-r_{0}^{2} V\left(\phi_{0}\right)}\left(r-r_{0}\right)+\cdots,
\end{aligned}
$$

where

$$
\begin{aligned}
f_{1}= & \frac{(n-2)(n-3)-r_{0}^{2} V\left(\phi_{0}\right)}{(n-2) r_{0}}, \\
f_{2}= & -\frac{1}{4(n-2)^{2} r_{0}^{3} f_{1}}\left[2(n-2)^{3}(n-3)^{2}-4(n-2)(n-3)^{2} r_{0}^{2} V\left(\phi_{0}\right)\right. \\
& \left.+2(n-4) r_{0}^{4} V\left(\phi_{0}\right)^{2}+3(n-2) r_{0}^{4} V^{\prime}\left(\phi_{0}\right)^{2}\right], \\
h_{2}= & -\frac{1}{4(n-2)^{2} r_{0}^{3} f_{1}^{2}}\left[2(n-2)^{3}(n-3)^{2}-4(n-2)(n-3)^{2} r_{0}^{2} V\left(\phi_{0}\right)\right. \\
& \left.+2(n-4) r_{0}^{4} V\left(\phi_{0}\right)^{2}-(n-2) r_{0}^{4} V^{\prime}\left(\phi_{0}\right)^{2}\right] .
\end{aligned}
$$

We can now use these inner Taylor expansions, either for the solitons or the black holes, to provide initial data for the numerical integration of the equations (2.10) out to infinity. Matching the asymptotic numerical results with the large- $r$ expansions we obtained previously, we can read off the parameters $\phi_{1}, \phi_{2}$ and the mass $M$ as functions of the free parameters of the inner expansion. Note that the parameter $h_{0}$ in the solitonic case, and the parameter $h_{1}$ in the black hole case, are "trivial" in the sense that they could be absorbed into a rescaling of the time coordinate. Thus $h_{0}$ or $h_{1}$ are not to be thought of as free parameters, but should instead be fixed by requiring that the asymptotic AdS metric has a canonically-normalised time coordinate. 
The upshot is that for the solitons, the inner solution has only one non-trivial free parameter, namely $\phi_{0}$. The mass $M$ and the scalar charges $\phi_{1}$ and $\phi_{2}$ in the asymptotic solutions are then all functions of $\phi_{0}$. This implies that we can, for example, view $M$ and $\phi_{2}$ as functions of $\phi_{1}$. For the black holes, the inner solutions are specified by two non-trivial parameters, namely the horizon radius $r_{0}$ and the parameter $\phi_{0}$. The three parameters $\left(M, \phi_{1}, \phi_{2}\right)$ in the asymptotic solutions are then determined as functions of these two inner parameters. With these data, we can then test the first law that we derived. We shall do this dimension by dimension from $D=4$ to $D=7$ in the following subsections. In all the numerical analysis, we set $\ell=1 / g=1$. Typically, we work to an accuracy of about three significant figures.

\section{$6.2 n=4$ dimensions}

For the $N=1$ potential, the Lagrangian is given by

$$
e^{-1} \mathcal{L}=R-\frac{1}{2}(\partial \phi)^{2}+6 g^{2} \cosh \left(\frac{1}{\sqrt{3}} \phi\right)
$$

The theory can be embedded in the STU supergravity model. The first law in this $\sigma=1$ example was discussed in section 5.1.1. (See also [10, 20].) Note that this potential is symmetric, and so in particular $\gamma_{3}=0$. Thus the "first law" for the soliton is given by

$$
d M=-\frac{1}{6} \phi_{2} d \phi_{1}+\frac{1}{12} \phi_{1} d \phi_{2} .
$$

In order to verify the above differential relation in the numerical solutions, it is convenient to make a Legendre transformation and define a new energy function $\widetilde{M}=M-\frac{1}{12} \phi_{1} \phi_{2}$ so that the first law becomes simply

$$
d \widetilde{M}=-\frac{1}{4} \phi_{2} d \phi_{1}
$$

As we discussed earlier, the soliton solution contains only one non-trivial parameter, and without loss of generality we may take this, in terms of the parameters in the asymptotic form of the solutions, to be $\phi_{1}$. Both $\widetilde{M}$ and $\phi_{2}$ are then functions of $\phi_{1}$. For small $\phi_{1} \lesssim 5$, we find that the numerical fits for $\widetilde{M}$ and $\phi_{2}$ are

$$
\begin{aligned}
\widetilde{M} & =0.0793 \phi_{1}^{2}+0.00436 \phi_{1}^{4}-0.000436 \phi_{1}^{5}+0.0000184 \phi_{1}^{6}+\cdots, \\
-\frac{1}{4} \phi_{2} & =0.158 \phi_{1}+0.0175 \phi_{1}^{3}-0.00221 \phi_{1}^{4}+0.000114 \phi_{1}^{5}+\cdots .
\end{aligned}
$$

Thus we see that the differential relation (6.11) is confirmed up to three significant figures.

Using the numerical methods, we can verify that scalar-charged black hole solutions also exist for (6.9). The asymptotic charges $\left(M, \phi_{1}, \phi_{2}\right)$ for these solutions are functions of the two inner parameters $r_{0}$ and $\phi_{0}$. The corresponding first law is given by

$$
d M=T d S-\frac{1}{6} \phi_{2} d \phi_{1}+\frac{1}{12} \phi_{1} d \phi_{2} .
$$


We shall make a specific choice for the value of $r_{0}$, namely $r_{0}=1$, which implies that the entropy is fixed and the first law is reduced to (6.11). We find that

$$
\begin{aligned}
\widetilde{M} & =1.00+0.0816 \phi_{1}^{2}+0.00436 \phi_{1}^{4}-0.000444 \phi_{1}^{5}+0.0000191 \phi_{1}^{6}, \\
-\frac{1}{4} \phi_{2} & =0.163 \phi_{1}+0.0175 \phi_{1}^{3}-0.00224 \phi_{1}^{4}+0.000118 \phi_{1}^{5} .
\end{aligned}
$$

Thus we see that (6.11) holds up to three significant figures. The Schwarzschild limit is achieved when the scalar charges vanish, and the resulting mass becomes $M=1=\widetilde{M}$ for $r_{0}=1$ and $g=1$. Note also that in this Taylor expansion of $\phi_{2}$ in terms $\phi_{1}$, the coefficient of $\phi_{1}^{2}$ vanishes. Of course our choice of a specific value for $r_{0}$ was not made without loss of generality (since we had already, without loss of generality, fixed the gauge coupling constant to $g=1$ ). In principle, we could repeat the computations for a range of different $r_{0}$ values, but here for the sake of brevity we selected just one choice.

We now consider the $N=2$ example, for which the Lagrangian is

$$
e^{-1} \mathcal{L}=R-\frac{1}{2}(\partial \phi)^{2}+2 g^{2}(\cosh \phi+2) .
$$

The theory can be embedded in either the STU model, or $\mathcal{N}=4$ gauged supergravity. Although the scalar potential is different from the $N=1$ case, we still have $\sigma=1$, and hence the first laws (6.10) and (6.13) are the same. The soliton and black hole solutions were constructed numerically in $[8,9]$. Following the same strategy as we did for the $N=1$ case, we find that for the soliton solution with $\phi_{2} \lesssim 2,\left(\widetilde{M}, \phi_{2}\right)$ can be expressed in terms of $\phi_{1}$ as follows

$$
\begin{aligned}
\widetilde{M} & =0.0796 \phi_{1}^{2}-0.00130 \phi_{1}^{4}-8.49 \times 10^{-6} \phi_{1}^{6}+\cdots, \\
-\frac{1}{4} \phi_{2} & =0.159 \phi_{1}-0.00520 \phi_{1}^{3}-0.0000504 \phi_{1}^{5} .
\end{aligned}
$$

For the black holes with $r_{0}=1$, we have

$$
\begin{aligned}
\widetilde{M} & =1.00+0.0819 \phi_{1}^{2}-0.00101 \phi_{1}^{4}+6.52 \times 10^{-6} \phi_{1}^{6}, \\
-\frac{1}{4} \phi_{2} & =0.164 \phi_{1}-0.00404 \phi_{1}^{3}+0.0000409 \phi_{1}^{5} .
\end{aligned}
$$

Thus for both cases, we find that the relation (6.11) holds to a good degree of precision.

Finally, we present in figure 1 plots of $\left(M, \phi_{2}\right)$ as functions of $\phi_{1}$ for larger ranges of $\phi_{1}$ for the soliton solutions.

\section{$6.3 n=5$ dimensions}

In $n=5$ dimensions, the scalar potentials become

$$
\begin{array}{ll}
N=1: & V=-4 g^{2}\left(2 e^{-\frac{1}{\sqrt{6}} \phi}+e^{\frac{2}{\sqrt{6}} \phi}\right), \\
N=2: & V=V_{N=1}(-\phi) .
\end{array}
$$



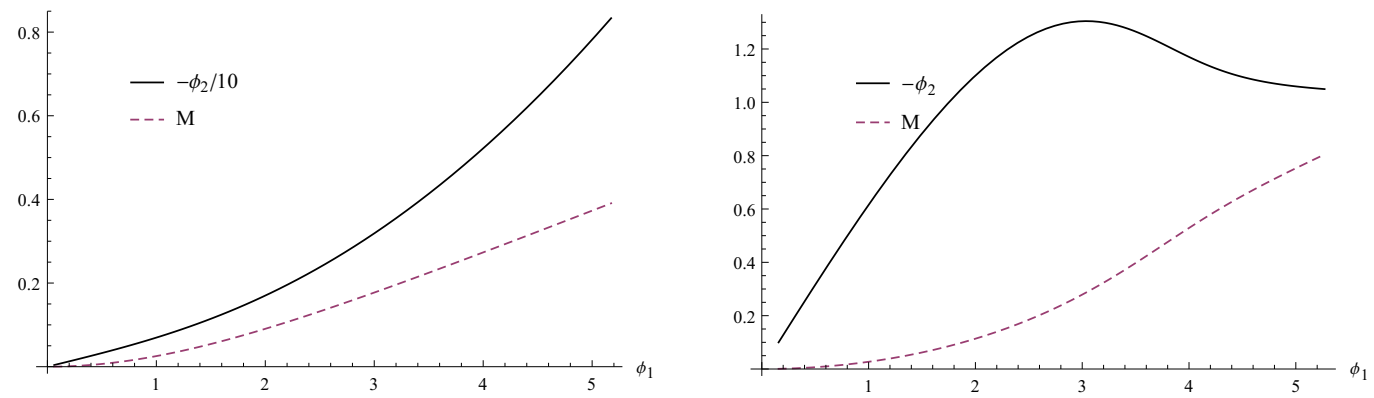

Figure 1. The left and right plots are for solitons in the $N=1$ and $N=2$ cases respectively. For $N=1,-\phi_{2}$ is a monotonically increasing function; we have verified this up to $\phi_{1} \sim 40$. For $N=2$, $-\phi_{2}$ has an extremum, which was observed also in [7].

Thus we need only consider the $N=1$ case. For this, we have $\sigma=0$, corresponding to the $\mathrm{BF}$ bound, for which the asymptotic behaviour for general dimensions was discussed in section 5.1.6. The first laws for solitons and black holes are given by

$$
\begin{aligned}
\text { Solitons : } \quad d M & =-\frac{\pi}{16}\left(\phi_{2} d \phi_{1}-\phi_{1} d \phi_{2}\right), \\
\text { Black holes : } & d M=T d S-\frac{\pi}{16}\left(\phi_{2} d \phi_{1}-\phi_{1} d \phi_{2}\right),
\end{aligned}
$$

where the mass $M$ is defined to be the holographic mass rather than the AMD mass, as discussed in section 5.1.6. For our purposes, we define $\widetilde{M}=M-\frac{\pi}{16} \phi_{1} \phi_{2}$, so that the first law for solitons becomes

$$
d \widetilde{M}=-\frac{\pi}{8} \phi_{2} d \phi_{1}
$$

For black holes, if we let the horizon radius $r_{0}$ and hence the entropy be fixed, the first law also reduces to (6.20). For solitons with small $\phi_{1} \lesssim 1$, we find

$$
\begin{aligned}
\widetilde{M} & =0.000686 \phi_{1}^{2}-0.0531 \phi_{1}^{3}-0.0178 \phi_{1}^{5}+\cdots, \\
-\frac{\pi}{8} \phi_{2} & =0.00141 \phi_{1}-0.160 \phi_{1}^{2}-0.0887 \phi_{1}^{4}+\cdots .
\end{aligned}
$$

For black holes with radius chosen again to be $r_{0}=1$, we find

$$
\begin{aligned}
\widetilde{M} & =2.36+0.00568 \phi_{1}^{2}-0.0497 \phi_{1}^{3}-0.0132 \phi_{1}^{5}+\cdots, \\
-\frac{\pi}{8} \phi_{2} & =0.0107 \phi_{1}-0.150 \phi_{1}^{2}-0.0667 \phi_{1}^{4}+\cdots .
\end{aligned}
$$

Thus we see that the relation (6.20) is well satisfied by both the solitons and by the black holes with the example value $r_{0}=1$. Note that the Schwarzschild AdS black hole arises when $\phi_{1}$ vanishes, corresponding to $M=\frac{3}{4} \pi=\widetilde{M}$.

\section{4 $n=6,7$ dimensions}

The scalar potentials are given in (6.4). In six dimensions, we have $\sigma=1$. The asymptotic behavior was discussed in [10], and also in section 4 of this paper. The first laws for the solitons and the black holes are given by

$$
\begin{aligned}
\text { Solitons : } & d M=-\frac{\pi}{30}\left(3 \phi_{2} d \phi_{1}-2 \phi_{1} d \phi_{2}\right), \\
\text { Black holes : } & d M=T d S-\frac{\pi}{30}\left(3 \phi_{2} d \phi_{1}-2 \phi_{1} d \phi_{2}\right) .
\end{aligned}
$$


For the $\sigma=1$ case, the holographic and AMD masses are both well defined, and they coincide. Defining $\widetilde{M}=M-\frac{\pi}{15} \phi_{1} \phi_{2}$, we find that the first laws for the solitons and black holes with fixed $r_{0}$ reduce to

$$
d \widetilde{M}=-\frac{\pi}{6} \phi_{2} d \phi_{1}
$$

The numerical analysis becomes more difficult to carry out for higher dimensions. For the $N=1,2$ cases, we find that (for $\phi_{1} \lesssim 1.5$ )

$N=1$ soliton:

$$
\begin{aligned}
\widetilde{M} & =0.392 \phi_{1}^{2}-0.0963 \phi_{1}^{4}+0.0498 \phi_{1}^{5}-0.0112 \phi_{1}^{6}+\cdots \\
-\frac{\pi}{6} \phi_{2} & =0.782 \phi_{1}-0.383 \phi_{1}^{3}+0.251 \phi_{1}^{4}-0.0695 \phi_{1}^{5}+\cdots
\end{aligned}
$$

$N=1$ black hole with $r_{0}=1$ :

$$
\begin{aligned}
\widetilde{M} & =4.19+0.391 \phi_{1}^{2}-0.0794 \phi_{1}^{4}+0.0348 \phi_{1}^{5}-0.00693 \phi_{1}^{6}+\cdots, \\
-\frac{\pi}{6} \phi_{2} & =0.781 \phi_{1}-0.313 \phi_{1}^{3}+0.169 \phi_{1}^{4}-0.0401 \phi_{1}^{5}+\cdots ;
\end{aligned}
$$

$N=2$ soliton:

$$
\begin{aligned}
\widetilde{M} & =0.421 \phi_{1}^{2}+0.127 \phi_{1}^{3}-0.00315 \phi_{1}^{5}+\cdots, \\
-\frac{\pi}{6} \phi_{2} & =0.843 \phi_{1}+0.379 \phi_{1}^{2}-0.0148 \phi_{1}^{4}+\cdots ;
\end{aligned}
$$

$N=2$ black hole with $r_{0}=1$ :

$$
\begin{aligned}
\widetilde{M} & =4.19+0.431 \phi_{1}^{2}+0.119 \phi_{1}^{3}-0.00200 \phi_{1}^{5}+\cdots, \\
-\frac{\pi}{6} \phi_{2} & =0.856 \phi_{1}+0.361 \phi_{1}^{2}-0.0101 \phi_{1}^{4}+\cdots .
\end{aligned}
$$

Thus we see that our numerical results show that the (6.24) is well satisfied. Note that the mass of the Schwarzschild AdS black hole with $r_{0}=1$ is $4 \pi / 3 \sim 4.19$.

In seven dimensions, we have $\sigma=2$, and the asymptotic behavior was discussed in section 5.1.5. The first law can be expressed as (5.33). In terms of $\widetilde{M}=M-\frac{\omega_{5}}{16 \pi} \phi_{1} \phi_{2}$, we have

We find for $\phi_{1} \lesssim 1$ that

$$
d \widetilde{M}=-\frac{\pi^{2}}{8} \phi_{2} d \phi_{1}
$$

$N=1$ soliton:

$$
\begin{aligned}
\widetilde{M} & =0.519 \phi_{1}^{3}-0.0788 \phi_{1}^{5}+\cdots, \\
-\frac{\pi^{2}}{8} \phi_{2} & =1.57 \phi_{1}^{2}-0.403 \phi_{1}^{4}+\cdots ;
\end{aligned}
$$

$N=1$ black hole with $r_{0}=1$ :

$$
\begin{aligned}
\widetilde{M} & =6.17+0.533 \phi_{1}^{3}-0.0688 \phi_{1}^{5}+\cdots, \\
-\frac{\pi^{2}}{8} \phi_{2} & =1.60 \phi_{1}^{2}-0.345 \phi_{1}^{4}+\cdots
\end{aligned}
$$


$N=2$ soliton:

$$
\begin{aligned}
\widetilde{M} & =0.0664 \phi_{1}^{2}+15.3 \phi_{1}^{3}-0.535 \phi_{1}^{4}+0.0542 \phi_{1}^{5}+\cdots, \\
-\frac{\pi^{2}}{8} \phi_{2} & =0.158 \phi_{1}+45.7 \phi_{1}^{2}-2.08 \phi_{1}^{3}+0.248 \phi_{1}^{4}+\cdots ;
\end{aligned}
$$

$N=2$ black hole with $r_{0}=1$ :

$$
\begin{aligned}
\widetilde{M} & =6.17+0.0719 \phi_{1}^{2}+15.3 \phi_{1}^{3}-0.542 \phi_{1}^{4}+0.0564 \phi_{1}^{5}+\cdots, \\
-\frac{\pi^{2}}{8} \phi_{2} & =0.164 \phi_{1}+45.7 \phi_{1}^{2}-2.11 \phi_{1}^{3}+0.258 \phi_{1}^{4}+\cdots .
\end{aligned}
$$

Thus we see that the relation (6.26) is well satisfied by both $N=1$ and $N=2$ solitons and black holes. Note that Schwarzschild black hole mass is $M=5 \pi^{2} / 8 \sim 6.17$ for $r_{0}=1$.

\section{Conclusions}

In this paper, we have studied some of the properties of static, spherically-symmetric, black hole and soliton solutions in $n$-dimensional theories of gravity coupled to a scalar field, in the case that there is a scalar potential $V(\phi)$ with a stationary point at $\phi=0$, with $V(0)<0$. This implies that there exist black hole and solitonic solutions that are asymptotic to anti-de Sitter spacetime.

Included amongst these solutions are AdS spacetime itself (the trivial "soliton") and the Schwarzschild-AdS black hole; in each of these cases the scalar field is everywhere zero. The solutions of interest to us in this paper are the ones where the scalar field is non-vanishing, and, therefore, dependent on the radial coordinate $r$. Provided the mass of the scalar lies in an appropriate range, these solutions are well-behaved and continue to approach anti-de Sitter spacetime at infinity. The scalar may, however, make a contribution to the mass of the black hole. More importantly, it makes a non-trivial contribution to the thermodynamics, providing an additional contribution in the first law, of the form given in (1.10).

The first law (1.10) was derived using the Wald procedure, which involves considering an infinitesimal variation of the parameters in a solution, and hence deriving a closed $(n-2)$ form whose integral $\delta \mathcal{H}$ over a bounding spacelike surface is therefore independent of deformations of the surface. This means in particular that $\delta \mathcal{H}_{\infty}=\delta \mathcal{H}_{H^{+}}$, where $\delta \mathcal{H}_{H^{+}}$ is evaluated on the outer horizon and $\delta \mathcal{H}_{\infty}$ is evaluated at infinity. For the metrics of interest, one finds $\delta \mathcal{H}_{H^{+}}=T \delta S$, while $\delta \mathcal{H}_{\infty}=\delta E+\left(c_{1} \phi_{2} \delta \phi_{1}-c_{2} \phi_{1} \delta \phi_{2}\right)$, hence leading to (1.10). Here $E$ is an integrable function of the parameters $\alpha, \phi_{1}$ and $\phi_{2}$ that characterise the asymptotic form of the solution, which is typically of the form (4.3). One may think of $E$ as the mass of the black hole, and in fact as we showed, it typically coincides with the mass calculated by means of the renormalised holographic stress tensor. In the black hole solutions, the three asymptotic parameters $\alpha, \phi_{1}$ and $\phi_{2}$ are actually (numerically) computable functions of the two non-trivial near-horizon parameters $r_{0}$ and $\phi_{0}$ that specify the family of black-hole solutions. 
It can be argued that if $\delta \mathcal{H}_{\infty}$ is non-integrable, then the concept of mass as the charge associated with a universally-defined and conserved Hamiltonian does not exist. However, one may still define the mass by other means, and as we saw, in the case of the Einsteinscalar black holes it can, in general at least, be defined via the AdS/CFT correspondence and the holographic stress tensor. The non-integrability of $\delta \mathcal{H}_{\infty}$ can then be attributed to the contribution of a term involving the scalar hair to the first law of thermodynamics. As we discussed in the introduction, one can construct other examples where $\delta \mathcal{H}_{\infty}$ is nonintegrable, such as charged black holes in Einstein-Maxwell theory when one works in a gauge where the electric potential vanishes on the horizon. In this example too, the nonintegrability is simply due to a contribution to the first law, namely the $\Phi d Q$ term in $d E=T d S+\Phi d Q$.

In our discussions we have considered three different ways to calculate the mass, or energy, of the Einstein-Scalar black holes. Firstly, as mentioned above, we used the renormalised holographic stress tensor of the dual field theory on the boundary of the asymptotically-AdS black hole spacetime. This method is capable of giving a finite answer in essentially all cases where the metric approaches the leading-order form of the AdS metric itself at large distances, although there can be complications in cases where there is a logarithmic dependence on the radial coordinate. There may also be ambiguities in the calculation, associated with the freedom to add a constant multiple of $\phi_{1} \phi_{2}$ to the energy. The second method we considered involves the use of the conformal technique developed by Ashtekar, Magnon and Das. This gives the mass as the integral of a certain electric component of the Weyl tensor at infinity. The AMD mass is finite provided that the metric approaches AdS no slower than in the Schwarzschild-AdS solution; that is to say, provided that the metric functions $h$ and $f$ have the form

$$
h(r)=r^{2} \ell^{-2}+1+\frac{\alpha}{r^{n-3}}+\cdots, \quad f(r)=r^{2} \ell^{-2}+1+\frac{\beta}{r^{n-3}}+\cdots,
$$

where the ellipses represent terms of higher order than those written.

The third way of calculating the mass that we discussed is from the Wald derivation of the first law. For the reasons we explained previously, one cannot simply interpret $\delta \mathcal{H}_{\infty}$ itself as the variation of the energy, because it contains an inherently non-integrable contribution from the variation of the parameters $\phi_{1}$ and $\phi_{2}$ in the asymptotic expansion of the scalar field. We can, however, separate off this non-intgerable contribution, and interpret the remainder of $\delta \mathcal{H}_{\infty}$ as the variation of the energy. This separation is not unique, but the non-uniqueness is the same as we saw before, namely the freedom to perform a Legendre transformation to a new energy function by adding a constant multiple of $\phi_{1} \phi_{2}$. This ambiguity can be fixed uniquely by defining one's choice of the relative ratio between the two terms in the non-integrable contribution $c_{1} \phi_{2} d \phi_{1}-c_{2} \phi_{1} d \phi_{2}$ in the first law.

Of the three methods for calculating the mass, the AMD procedure is the least widely applicable. However, in cases where it can be applied, it provides results that are consistent with the other two. The holographic mass calculation and the calculation from the first law in general yield consistent results, in cases where they can both be applied. We found one example, in seven dimensions, where we could only remove a logarithmic divergence in 
the holographic mass by the rather questionable introduction of a non-local counterterm. In this example, by contrast, the mass could still be calculated by our third method, via the first law.

There is also a question as to whether one should allow variations of $\phi_{1}$ and $\phi_{2}$ in a "first law" that correspond to making changes to the boundary conditions of the scalar field. This seems to be more a question of viewpoint rather than of substance. In his derivation, Wald distinguishes between two versions of the first law, namely the "physical states version" and the "equilibrium states version" [3]. In the former, one envisages an actual physical process by which a stationary black hole evolves into a new final stationary black hole state. In the latter, one simply starts from a given stationary solution and compares it with a nearby solution obtained by making infinitesimal variations of the parameters in the solution. We are taking this latter viewpoint in our discussion, and the first law (1.10) can be taken to be simply a mathematical statement of how the entropy, viewed as a function of the parameters specifying the black hole, changes under infinitesimal variations of those parameters. The formula is valid whether one restricts to variations that preserve the asymptotic boundary conditions on the scalar field or not. ${ }^{6}$

In fact, we have argued that one more or less has to adopt such a viewpoint when considering black holes in a system such as the Einstein-Scalar theory that we have studied in this paper. There have been discussions in the past, such as in $[8,18]$, where solitonic solutions in the Einstein-Scalar theory have been considered. For these solutions one can see that there exists a functional relationship between the parameters $\phi_{1}$ and $\phi_{2}$ in the asymptotic expansion of the scalar field, and so one can integrate up the entire right-hand side in the expression (1.6). Thus it could be absorbed into a redefinition of the mass, thereby sidestepping the need to view the $c_{1} \phi_{2} d \phi_{1}-c_{2} \phi_{1} d \phi_{2}$ terms as a distinct and separate contribution in the first law. However, this is a somewhat restricted conclusion, resulting from looking at non-generic solutions in the theory. For the black holes, as opposed to the solitons, there is an additional parameter in the solutions, $\phi_{1}$ can no longer be viewed as a function only of $\phi_{2}$, and so the $c_{1} \phi_{2} \delta \phi_{1}-c_{2} \phi_{1} \delta \phi_{2}$ terms in (1.6) cannot be integrated up and absorbed into a redefinition of the mass. A concrete and fully explicit example of this kind is provided by the dyonic Kaluza-Klein AdS black hole constructed in [20]. In these circumstances, it becomes natural to adopt the equilibrium states interpretation, and include the $c_{1} \phi_{2} d \phi_{1}-c_{2} \phi_{1} d \phi_{2}$ terms as a distinct additional contribution in the first law. By this means one obtains a first law (1.10) for the Einstein-Scalar black holes whose right-hand side is an exact differential in the two-dimensional parameter space of solutions, leading to an integrable energy function $E$. As we showed, this energy function is in agreement with the AMD or the holographic mass, in situations where those calculations are well defined.

\footnotetext{
${ }^{6} \mathrm{~A}$ somewhat analogous example of a situation where one allows variations outside those usually considered is in a theory such as Einstein gravity with a cosmological constant, where the cosmological constant itself is allowed to vary, and is treated as a further thermodynamic variable having an interpretation as a pressure (see, for example, [34, 35]). That example is in a sense more extreme, in that one is actually treating a parameter in the Lagrangian as a thermodynamic variable. Nevertheless, one can explore the mathematical consequences of allowing such variations in the space of solutions, and one thereby derives new insights into the concept of a conjugate "thermodynamic volume."
} 


\section{Acknowledgments}

We are grateful to Sijie Gao, Hai-shan Liu, Yi Pang, Harvey Reall, Kostas Skenderis, David Tong and Bob Wald for helpful discussions. We gratefully acknowledge the hospitality of the KITPC, Beijing, where much of this work was carried out. The research of H.L. is supported in part by NSFC grants 11175269 and 11235003 . The work of C.N.P. is supported in part by DOE grant DE-FG02-13ER42020. W.Q. is supported in part by NSFC grant 11135006 .

Open Access. This article is distributed under the terms of the Creative Commons Attribution License (CC-BY 4.0), which permits any use, distribution and reproduction in any medium, provided the original author(s) and source are credited.

\section{References}

[1] R.M. Wald, Black hole entropy is the Noether charge, Phys. Rev. D 48 (1993) 3427 [gr-qc/9307038] [INSPIRE].

[2] V. Iyer and R.M. Wald, Some properties of Noether charge and a proposal for dynamical black hole entropy, Phys. Rev. D 50 (1994) 846 [gr-qc/9403028] [INSPIRE].

[3] R.M. Wald, Quantum Field Theory in Curved Spacetime and Black Hole Thermodynamics, University of Chicago Press, Chichago, U.S.A. (1994).

[4] M. Henneaux, C. Martinez, R. Troncoso and J. Zanelli, Black holes and asymptotics of $2+1$ gravity coupled to a scalar field, Phys. Rev. D 65 (2002) 104007 [hep-th/0201170] [INSPIRE].

[5] M. Henneaux, C. Martinez, R. Troncoso and J. Zanelli, Asymptotically anti-de Sitter spacetimes and scalar fields with a logarithmic branch, Phys. Rev. D 70 (2004) 044034 [hep-th/0404236] [INSPIRE].

[6] M. Henneaux, C. Martinez, R. Troncoso and J. Zanelli, Asymptotic behavior and Hamiltonian analysis of anti-de Sitter gravity coupled to scalar fields, Annals Phys. 322 (2007) 824 [hep-th/0603185] [INSPIRE].

[7] T. Hertog and K. Maeda, Black holes with scalar hair and asymptotics in $N=8$ supergravity, JHEP 07 (2004) 051 [hep-th/0404261] [INSPIRE].

[8] T. Hertog and G.T. Horowitz, Designer gravity and field theory effective potentials, Phys. Rev. Lett. 94 (2005) 221301 [hep-th/0412169] [INSPIRE].

[9] T. Hertog and G.T. Horowitz, Holographic description of AdS cosmologies, JHEP 04 (2005) 005 [hep-th/0503071] [INSPIRE].

[10] H.-S. Liu and H. Lü, Scalar Charges in Asymptotic AdS Geometries, Phys. Lett. B 730 (2014) 267 [arXiv:1401.0010] [INSPIRE].

[11] V. Balasubramanian and P. Kraus, A stress tensor for Anti-de Sitter gravity, Commun. Math. Phys. 208 (1999) 413 [hep-th/9902121] [INSPIRE].

[12] R.C. Myers, Stress tensors and Casimir energies in the AdS/CFT correspondence, Phys. Rev. D 60 (1999) 046002 [hep-th/9903203] [INSPIRE].

[13] R. Emparan, C.V. Johnson and R.C. Myers, Surface terms as counterterms in the AdS/CFT correspondence, Phys. Rev. D 60 (1999) 104001 [hep-th/9903238] [INSPIRE].

[14] S. de Haro, S.N. Solodukhin and K. Skenderis, Holographic reconstruction of space-time and renormalization in the AdS/CFT correspondence, Commun. Math. Phys. 217 (2001) 595 [hep-th/0002230] [INSPIRE]. 
[15] P. Kraus, F. Larsen and R. Siebelink, The gravitational action in asymptotically AdS and flat space-times, Nucl. Phys. B 563 (1999) 259 [hep-th/9906127] [INSPIRE].

[16] A. Ashtekar and A. Magnon, Asymptotically anti-de Sitter space-times, Class. Quant. Grav. 1 (1984) L39 [INSPIRE].

[17] A. Ashtekar and S. Das, Asymptotically Anti-de Sitter space-times: Conserved quantities, Class. Quant. Grav. 17 (2000) L17 [hep-th/9911230] [INSPIRE].

[18] A. Anabalón, D. Astefanesei and C. Martinez, Mass of asymptotically anti-de Sitter hairy spacetimes, Phys. Rev. D 91 (2015) 041501 [arXiv:1407.3296] [InSPIRE].

[19] W. Chen, H. Lü and C.N. Pope, Mass of rotating black holes in gauged supergravities, Phys. Rev. D 73 (2006) 104036 [hep-th/0510081] [INSPIRE].

[20] H. Lü, Y. Pang and C.N. Pope, AdS Dyonic Black Hole and its Thermodynamics, JHEP 11 (2013) 033 [arXiv: 1307.6243] [inSPIRE].

[21] D.D.K. Chow and G. Compère, Dyonic AdS black holes in maximal gauged supergravity, Phys. Rev. D 89 (2014) 065003 [arXiv:1311.1204] [InSPIRE].

[22] S. Gao, The first law of black hole mechanics in Einstein-Maxwell and Einstein-Yang-Mills theories, Phys. Rev. D 68 (2003) 044016 [gr-qc/0304094] [inSPIRE].

[23] H.-S. Liu, H. Lü and C.N. Pope, Thermodynamics of Einstein-Proca AdS Black Holes, JHEP 06 (2014) 109 [arXiv: 1402.5153] [INSPIRE].

[24] K. Hotta, Y. Hyakutake, T. Kubota, T. Nishinaka and H. Tanida, The CFT-interpolating Black Hole in Three Dimensions, JHEP 01 (2009) 010 [arXiv:0811.0910] [INSPIRE].

[25] A. Anabalón, Exact Black Holes and Universality in the Backreaction of non-linear $\sigma$-models with a potential in (A)dS $S_{4}$, JHEP 06 (2012) 127 [arXiv:1204.2720] [INSPIRE].

[26] A. Anabalón, D. Astefanesei and R. Mann, Exact asymptotically flat charged hairy black holes with a dilaton potential, JHEP 10 (2013) 184 [arXiv:1308.1693] [INSPIRE].

[27] A. Anabalón and D. Astefanesei, On attractor mechanism of AdS 4 black holes, Phys. Lett. B 727 (2013) 568 [arXiv: 1309.5863] [INSPIRE].

[28] P.A. González, E. Papantonopoulos, J. Saavedra and Y. Vásquez, Four-Dimensional Asymptotically AdS Black Holes with Scalar Hair, JHEP 12 (2013) 021 [arXiv:1309.2161] [INSPIRE].

[29] A. Aceña, A. Anabalón, D. Astefanesei and R. Mann, Hairy planar black holes in higher dimensions, JHEP 01 (2014) 153 [arXiv: 1311.6065] [INSPIRE].

[30] X.-H. Feng, H. Lü and Q. Wen, Scalar Hairy Black Holes in General Dimensions, Phys. Rev. D 89 (2014) 044014 [arXiv: 1312.5374] [INSPIRE].

[31] H. Lü, Charged dilatonic AdS black holes and magnetic $A d S_{D-2} \times R^{2}$ vacua, JHEP 09 (2013) 112 [arXiv: 1306.2386] [INSPIRE].

[32] S.-Q. Wu, General rotating charged Kaluza-Klein AdS black holes in higher dimensions, Phys. Rev. D 83 (2011) 121502 [arXiv:1108.4157] [INSPIRE].

[33] D.D.K. Chow, Two-charge rotating black holes in four-dimensional gauged supergravity, Class. Quant. Grav. 28 (2011) 175004 [arXiv: 1012.1851] [INSPIRE].

[34] D. Kastor, S. Ray and J. Traschen, Enthalpy and the Mechanics of AdS Black Holes, Class. Quant. Grav. 26 (2009) 195011 [arXiv:0904.2765] [INSPIRE].

[35] M. Cvetič, G.W. Gibbons, D. Kubiznak and C.N. Pope, Black Hole Enthalpy and an Entropy Inequality for the Thermodynamic Volume, Phys. Rev. D 84 (2011) 024037 [arXiv: 1012.2888] [INSPIRE]. 